\title{
What Can We Learn about Magnetotail Reconnection from 2D PIC Harris-Sheet Simulations?
}

\author{
M.V. Goldman ${ }^{1}$ - D.L. Newman ${ }^{1}$ - G. Lapenta ${ }^{2}$
}

Received: 1 October 2014 / Accepted: 28 April 2015 / Published online: 26 May 2015

(C) The Author(s) 2015. This article is published with open access at Springerlink.com

\begin{abstract}
The Magnetosphere Multiscale Mission (MMS) will provide the first opportunity to probe electron-scale physics during magnetic reconnection in Earth's magnetopause and magnetotail. This article will address only tail reconnection-as a non-steady-state process in which the first reconnected field lines advance away from the $x$-point in flux pile-up fronts directed Earthward and anti-Earthward. An up-to-date microscopic physical picture of electron and ion-scale collisionless tail reconnection processes is presented based on 2-D Particle-In-Cell (PIC) simulations initiated from a Harris current sheet and on Cluster and Themis measurements of tail reconnection. The successes and limitations of simulations when compared to measured reconnection are addressed in detail. The main focus is on particle and field diffusion region signatures in the tail reconnection geometry. The interpretation of these signatures is vital to enable spacecraft to identify physically significant reconnection events, to trigger meaningful data transfer from MMS to Earth and to construct a useful overall physical picture of tail reconnection. New simulation results and theoretical interpretations are presented for energy transport of particles and fields, for the size and shape of electron and ion diffusion regions, for processes occurring near the fronts and for the $\mathbf{j} \times \mathbf{B}$ (Hall) electric field.
\end{abstract}

Keywords MMS · Reconnection · Magnetotail · Transport · Front · Diffusion region

\section{Introduction}

Scope of Paper Improved understanding of magnetic reconnection in Earth's magnetotail is a multi-faceted subject which requires answers to at least the following three questions:

(a) How does reconnection begin? (i.e., the onset problem: how is an $x$-point formed?)

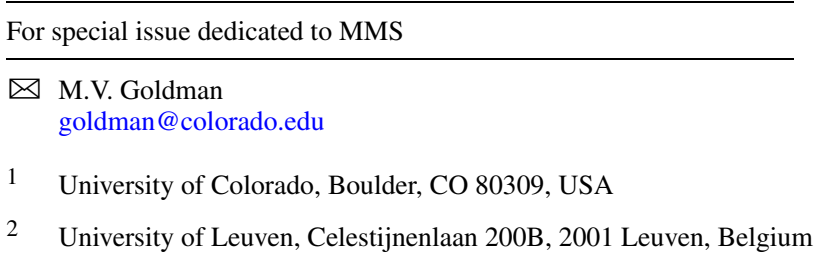


(b) How can fields, waves and particles in the outflow exhaust and separatrix be characterized? (i.e., How are diffusion regions defined and where are they located?)

(c) What are the properties of magnetic flux pile-up fronts propagating Earthward and antiEarthward from an $x$-point in the tail? (i.e., Are pile-up fronts the same as dipolarization fronts? How do fronts contribute to positive and negative work controlling energization of the tail?)

Collisionless Particle-In-Cell (PIC) simulations in two and three dimensions with various drivers, boundary and initial conditions have informed all of these as well as other questions about tail reconnection. Although the present paper addresses a number of these questions, it is neither a review of magnetotail reconnection, nor is it a review of all PIC simulations relevant to tail reconnection.

The principal object of this paper is to demonstrate what can be learned about diffusion regions in Earth's magnetotail (i.e., question (b) above) from 2D PIC Harris-current-sheet reconnection simulations that is relevant to NASA's Magnetospheric MultiScale (MMS) mission. The main goal for MMS, as stated by NASA (http://mms.gsfc.nasa.gov/science.html), is the following: "The overarching goal of the MMS mission is to measure the plasma and the electric and magnetic fields inside the diffusion regions of Earth's magnetosphere...".

The many different (and often subtle) theoretical signatures of diffusion regions are reviewed and developed. These will be useful in the identification of significant future MMS events and in the vetting of a wide range of magnetotail and magnetopause simulations.

The directions and magnitude of electric and magnetic fields (in $\mathrm{mV} / \mathrm{m}$ and $\mathrm{nT}$ ) are extracted from simulations with artificial mass ratios by making either the electrons or the ions physical, depending on the relevant physics. These electric and magnetic fields often agree not only qualitatively but quantitatively with measurements. For example, (Hall) electric and magnetic field strengths found in the simulations agree quantitatively with corresponding fields measured during tail reconnection (Wygant et al. 2005; Eastwood et al. 2010a, 2010b).

In order to elucidate the implications of using $\mathbf{J} \cdot \mathbf{E}$ work as a signature of diffusion regions the present paper also investigates transport of particle and magnetic energy in detail for the 2D Harris-sheet simulations.

In addition to diffusion region studies, a few (but not all) measurements of magnetic flux pile-up fronts (question (c) above) are addressed and found to be either qualitatively or quantitatively well-described by 2D PIC simulations with initial 1D Harris sheets. Examples include Themis observations (Runov et al. 2009) of the thickness of the DF, the strength of the piled-up normal (GSM-z) magnetic field, the local reconnection electric field (Eastwood et al. 2015), the density profile at the front and the competition between particle and magnetic pressure at the front.

However a number of other key pile-up front features, such as plasmoid formation, bubbles, interchange instabilities, and front precursor effects (Angelopoulos et al. 2013; Zhou et al. 2013) are not seen in simulations with a simple Harris current sheet because they require simulations in three dimensions (Vapirev et al. 2013) and/or because they require initial current sheets which are nonuniform in two-dimensions, such as Lembege-Pellat equilibria (Lembege and Pellat 1982; Sitnov et al. 2009). Additional important simulations which are either 3D or employ 2-D inhomogeneous current sheets (or both) are: Pritchett (2005b, 2010); Pritchett and Coroniti (2011, 2013); Sitnov et al. (2013); Birn and Hesse (2014); and Bessho and Bhattacharjee (2014).

Issues concerning reconnection onset (question (a), above) are still controversial. Theory and simulations show that X-points can be created on current sheet by a variety of possible tearing instabilities (Coppi et al. 1966; Drake and Lee 1977; Bulanov et al. 1992; Sitnov 
and Schindler 2010). Distinctions between spontaneous and driven reconnection are often blurred and unresolved. It is not known whether or not the nature and properties of evolved diffusion regions are sensitive to the details of reconnection onset.

The Harris current sheet simulations presented in this paper use a "seed perturbation" (Markidis and Lapenta 2010)—an expeditious but artificial device for "driving" electron tearing instabilities which lead to an evolved reconnection geometry which is here studied in detail. The correct physics of reconnection onset in this paper is left unresolved. This is consistent with other recent studies which have been used to produce outflow and pile-up fronts without addressing the onset problem at all (Liu et al. 2012).

Past 2D PIC Harris-Sheet Reconnection Simulations Relevant to Observations 2D PIC reconnection simulations starting with Harris current sheets have been used successfully by many observers, often in collaboration with theorists and simulators, to explain magnetotail reconnection measurements. To list just a few:

- Bipolar electric fields interpreted as electron phase space holes during tail reconnection in the vicinity of the separatrix (Cattell et al. 2005)

- Hall magnetic field distortions due to magnetic guide-fields based on magnetotail observations (Eastwood et al. 2010a)

- A statistical survey of 33 tail reconnection events (Eastwood et al. 2010b)

- Hall fields and ion reflection and acceleration found by Themis in the vicinity of dipolarization fronts and compared favorably with Harris sheet PIC simulations by the authors of this paper (Eastwood et al. 2015)

2D Harris sheet reconnection simulations have been used as well in early work which recognized the importance of Hall physics and fields (Drake et al. 2008). More recently they have been used to describe agyrotropy in the electron distribution functions in the electron diffusion region (Scudder and Daughton 2008; Aunai et al. 2013). They have also been used recently to predict intense fast Poynting flux propagation away from the $x$-point along separatrices which have been interpreted as one possible origin of the intense Poynting flux which eventually enters the auroral ionosphere (Shay et al. 2011). Current new work in progress by the authors in collaboration with Dr. J. Eastwood of Imperial College has shown electron temperature anisotropy behind dipolarization fronts and nearby whistler waves and electron phase space holes in 2D Harris simulations corresponding well with satellite observations of these features (Deng et al. 2010; Khotyaintsev et al. 2011).

\subsection{Physical Picture of Tail Reconnection from 2-D Simulations and Measurements}

Tail reconnection is thought to occur where the cross-tail current sheet thins down considerably from its normal range of thicknesses ( 1 to 3 Earth radii, $R_{E}$ ) to less than an ion inertial length, $d_{i}=c / \sqrt{\left(4 \pi e^{2} n_{i} / M_{i}\right)}$, so that tearing instabilities can take place. For a nominal value $R_{E}=10 d_{i}\left(n_{i}=0.13 / c c\right)$ this represents thinning by a factor of 20 to 60 .

A very simplified initial equilibrium containing the already-thinned cross-tail current sheet is often employed in kinetic simulations of symmetric tail reconnection. This uniform thin current sheet separates oppositely-directed straight magnetic field line components which asymptote to a constant magnetic field, $B_{0}$, far from the current sheet. The already- 
thinned current sheet is frequently modeled in simulations by a Harris sheet of uniform thickness $d_{i} / 2$ and length (in GSM-x) of several hundred $d_{i}$. By contrast, the physical ambient cross-tail current sheet is much thicker than $d_{i} / 2$, except possibly in a narrow region where an $x$-point forms. The greater thickness away from the $x$-point has important physical consequences for processes such as dipolarization front propagation and transport which have yet to be modeled properly in simulations, including those reported here. In collisionless simulations the Harris equilibrium is kinetic (Harris 1962; Yamada et al. 2000, 2010), with 1-D spatial variation of the density in the GSM-y-direction orthogonal to the current sheet plane and a purely diamagnetic current carried mainly by the hotter species (usually ions). The peak density of the Harris current sheet, $n_{0}$, is used to define an ion-inertial length unit, $d_{i 0} \approx 230 \mathrm{~km}$ for physical ions and density $n_{0}=1 / c c$. In the usually-employed initial Harris current sheet there is no electric field and the assumed initial conditions determine the ratio $B_{0} / \sqrt{n_{0}}$ through the balance between magnetic and particle pressures. This ratio is proportional to the ratio of the Harris Alfven speed, $v_{A 0}$ to the speed of light, $c$, which is the same as the ratio, $\Omega_{i 0} / \omega_{i}$ of the ion cyclotron frequency, $\Omega_{i 0}=e B_{0} / M_{i} c$ to the Harris ion plasma frequency, $\omega_{i}=\sqrt{\left(4 \pi n_{0} e^{2} / M_{i}\right)}$. The ratio $v_{A 0} / c$ can be expressed in terms of the initial temperature ratio $T_{i} / T_{e}$ (nominally $=5$ ), the assumed mass ratio, $m_{e} / M_{i}$, and the initial electron thermal velocity, $v_{e}=\sqrt{\left(T_{e} / m_{e}\right)}$ (nominally $\left.=0.045\right)$ in units of the speed of light, $c$ :

$$
\begin{aligned}
& \frac{v_{A 0}}{c}=\frac{B_{0}}{\sqrt{\left(4 \pi n_{0} M_{i} c^{2}\right)}}=\frac{v_{e}}{c} \sqrt{\frac{m_{e}}{M_{i}}} \sqrt{2} \sqrt{\left(1+T_{i} / T_{e}\right)} \\
& \text { For } M_{i} / m_{e}=1836, \quad \frac{c}{v_{A 0}} \approx \sqrt{2} \times 10^{3}\left(\frac{10^{-8} \text { Tesla }}{B_{0}}\right) \times \sqrt{\frac{n_{0}}{1 \mathrm{~cm}^{-3}}}
\end{aligned}
$$

It is evident from Eq. (1a) that the simulation input parameters determine the ratio $B_{0} / \sqrt{n_{0}}$, so that when comparing with observations, either $B_{0}$ or $n_{0}$ can be specified but not both independently. The second equation, (1b) can be used with spacecraft measurements of $B_{0}$ and $n_{0}$ separately to evaluate the physical ratio of $c / v_{A 0}$ for physical ions.

When the current sheet thickness is on the order of the ion skin depth $\left(c / \omega_{i}\right)$ or less, the Harris equilibrium is unstable to spontaneous reconnection through collisionless tearing instabilities that create $x$-points. Coppi et al. (1966) derived the growth rate of electron tearing modes for a Harris current sheet and applied it to the magnetotail. Simulations show that after tearing, the magnetic tension in newly reconnected flux tubes makes them snap away from the $x$-point with the outflow exhaust. The resulting out-of-plane induction (reconnection) electric field causes inflow of unreconnected field lines, which are topologically converted into reconnected field lines at the $x$-point. However, there is relatively little energy exchange between the magnetic field and the plasma in the vicinity of the $x$-point.

Tearing instabilities are often very slow (typical growth rates for tail parameters are on the order of 10's of ion inverse cyclotron frequencies, $\Omega_{c i}^{-1}$ ). Kinetic simulations of spontaneous reconnection initiated solely by this instability have been performed (Pritchett 2005a), but they are time-consuming. To speed things up, simulations of kinetic reconnection are usually driven, either by an initial perturbation which effectively introduces a very small local normal magnetic field across the thin current sheet (Lapenta et al. 2011) or by an $E \times B$ drift in the inflow, which temporally thins the current sheet and speeds up the tearing (Daughton et al. 2011).

A uniform background population (from lobe or plasmasheet particles sandwiching the cross-tail current sheet modeled by the Harris sheet) may be added to the Harris equilibrium 
without affecting its self-consistency. The background density is usually taken to be a density, $n_{b}$, lower than the Harris density, $n_{0}$. The background density is dynamically significant in Harris sheet simulations because as the Harris sheet "unzips" after tearing it is replaced by background electrons, some of which form a replacement electron current sheet at the $x$-line and on the nearby legs of the separatrix. Before heating occurs there is a pressure difference due to the downstream positive density gradient behind and at the first reconnected field lines, which exerts a force opposite to the magnetic tension pulling magnetic field lines away from the $x$-point. This creates a flux pile-up "front" of the (mainly) GSM-z component of the first reconnected field lines, which initially are slowed down relative to the reconnected field lines upstream. The flux front is called a dipolarization front (DF) when Earth's dipolar field is also modeled in the simulation (Sitnov et al. 2009). For our purposes we will call it the flux pile-up front or simply the front. Measurements suggest that the ionoutflow velocity upstream of the front is moving faster than the front, whereas the reverse is found in many PIC simulations. Recent special simulations (Liu et al. 2012) based on the so-called Riemann problem avoid initial value issues and do not employ two densities. In these simulations the ion-outflow-velocity behind the front is found to be faster than the front. It is also faster than the front in new 3D Riemann-related simulations (Drake et al. 2014).

Initial-value PIC simulations which start from a Harris sheet and lower density background have been used extensively for treating non-steady-state reconnection in which a moving front is an essential part of the reconnection geometry. In fact, most reconnection events in the tail may be of this bursty nature, rather than steady-state (A. Runov, private communication), although there are exceptions in which tail reconnection can last as long as an hour (H. Hietel, private communication). Fronts appear to play an important role in energy exchange between particles and fields (Hamrin et al. 2012; Angelopoulos et al. 2013; Zhou et al. 2013; Lapenta et al. 2014; Vapirev et al. 2013) and may be more energetically significant than the $x$-line or electron diffusion region, which governs the rate of topological transformation from pre-reconnected to post-reconnected magnetic field lines.

A uniform out-of-plane guide field, $B_{g}$, (in the GSM-y direction) may be added without affecting the self-consistency of the Harris equilibrium. In the majority of measured tail reconnection events characterized as anti-parallel (Eastwood et al. 2010a, 2010b) a small guide field, $B_{g} \leq 1 \mathrm{nT}$ may still be present (Eastwood, private communication). However simulations show that a guide field of $B_{g} \leq 1 \mathrm{nT}$ can have significant measurable consequences (Goldman et al. 2011; Le et al. 2013) which are missing from antiparallel reconnection simulations in which $B_{g}$ is take as strictly zero. In most of the implicit PIC simulations described in this paper the ion to electron mass-ratio is taken to be $M_{i} / m_{e}=256$ and the guide field is taken to be $B_{g}=0.1 B_{0}$. The (artificial) mass-ratio employed in simulations is significant because the effect of a guide field, $B_{g}$, on electronscale reconnection features depends on the mass-ratio, and are pronounced even for very small $B_{g}$ in simulations with a physical mass ratio, $M_{i} / m_{e}=1836$ (Lapenta et al. 2011; Goldman et al. 2011). One example is the deflection of electron-jets associated with the socalled external diffusion region seen in strictly anti-parallel simulations with $M_{i} / m_{e}$ ratios as low as 25 (Shay et al. 2007). The critical $B_{g}$ for electron-jet deflection depends on mass ratio as $\left[B_{g} / B_{0}\right]_{\text {crit }} \propto \sqrt{\left(m_{e} / M_{i}\right)}$ (Le et al. 2013) and is consistent with earlier studies using both physical and artificial mass ratios (Goldman et al. 2011). Hence, as far as electronscale processes are concerned, the assumed guide field $B_{g}=0.1 B_{0}$ in simulations described in this paper has the same effect as $B_{g}=0.04 B_{0}$ for a physical mass ratio, $M_{i} / m_{e}=1836$, and is equivalent to $B_{g}=0.3 B_{0}$ in a number of other simulations employing a mass ratio, $M_{i} / m_{e}=25$. In tail reconnection $B_{0}$ has been measured to be 10-30 nT (Cattell et al. 2005; 
Runov et al. 2009) implying a critical guide field from $0.4 \mathrm{nT}$ to $1 \mathrm{nT}$. One important effect of such small initial guide fields on tail reconnection is the elimination of the long thin external electron jets and associated external diffusion region found in antiparallel simulations (Goldman et al. 2011).

\subsection{Simulations}

Simulation coordinates, $x, y$ and $z$ will be used in describing simulation results. Table 1 relates simulation coordinates to GSM coordinates. Henceforth, any unlabeled coordinates will always be simulation coordinates. GSM coordinates will always be denoted by GSM.

Artificial mass ratios are used to speed up reconnection simulations which often need to employ large simulation boxes and run for long physical times. However significant progress has been made in recent years towards using physical mass ratios in reconnection simulations (Lapenta et al. 2010, 2011; Markidis et al. 2011; Goldman et al. 2011; Le et al. 2013).

The use of artificial mass ratios requires careful interpretation of results. For example, in simulations with $M_{i} / m_{e}=256$ one can take either $m_{e}$ or $M_{i}$ to be physical, but not both. In the study of electron-scale processes, $m_{e}$ should be taken as the physical mass so the ions must be interpreted as lighter than 1836 times the electron mass. However, in the study of ion-scale processes, $M_{i}$ is taken as the physical mass and the electrons will be interpreted as heavier than normal $\left(m_{e}=M_{i} / 256\right.$ instead of $\left.m_{e}=M_{i} / 1836\right)$.

In the implicit 2D PIC simulations starting from a Harris equilibrium to be described below the input parameters are the Harris-sheet thickness, $\Delta$, the mass ratio, $M_{i} / m_{e}$, the electron thermal velocity, $v_{e} / c$, the electron to ion temperature ratio, $T_{e} / T_{i}$, the background to Harris density, $n_{b} / n_{0}$ and the ratio of guide to asymptotic magnetic field, $B_{g} / B_{0}$. Values for these input parameters for what we will call simulation "A" are given in Table 2, together with the simulation box size and the effective electron and ion temperatures for the two different interpretations of the artificial mass ratio.

Table 1 Coordinate systems

\begin{tabular}{ll}
\hline Simulation coordinates & GSM coordinates \\
\hline$x$ & $x_{\mathrm{GSM}}$ \\
$y$ & $z_{\mathrm{GSM}}$ \\
$z$ & $-y_{\mathrm{GSM}}$ \\
\hline
\end{tabular}

Table 2 Simulation A, physical electron mass

\begin{tabular}{lllll}
\hline $\begin{array}{l}\text { Input params } \\
\text { for Simulation A }\end{array}$ & Value & $\begin{array}{l}\text { If } m_{e} \text { is take } \\
\text { as physical }\end{array}$ & $\begin{array}{l}\text { If } M_{i} \text { is taken } \\
\text { as physical }\end{array}$ & $\begin{array}{l}\text { Derived from } \\
\text { input params }\end{array}$ \\
\hline $\begin{array}{l}\left.L_{x} \times L_{y} \text { (units of } d_{i 0}\right) \\
\Delta / d_{i 0}\end{array}$ & $\begin{array}{l}200 \times 30 \\
0.5\end{array}$ & & & \\
$M_{i} / m_{e}$ & 256 & & & \\
$v_{e} / c$ & 0.045 & $T_{e}=1 \mathrm{keV}$ & $T_{e}=7 \mathrm{keV}$ & $c / v_{A 0}=103$ \\
$T_{i} / T_{e}$ & 5 & $T_{i}=5 \mathrm{keV}$ & $T_{i}=35 \mathrm{keV}$ & $\begin{array}{l}n_{0}=0.05 \mathrm{~cm}^{-3} \\
d_{i 0}=1000 \mathrm{~km} \\
\text { for } B_{0}=30 \mathrm{nT} \\
n_{b} / n_{0}\end{array}$ \\
$B_{g} / B_{0}$ & 0.1 & & & $c / v_{A b}=33$ \\
\hline
\end{tabular}


Table 3 Simulation B, physical ion mass

\begin{tabular}{lllll}
\hline $\begin{array}{l}\text { Input params } \\
\text { for Simulation B }\end{array}$ & Value & $\begin{array}{l}\text { If } m_{e} \text { is take } \\
\text { as physical }\end{array}$ & $\begin{array}{l}\text { If } M_{i} \text { is taken } \\
\text { as physical }\end{array}$ & $\begin{array}{l}\text { Derived from } \\
\text { input params }\end{array}$ \\
\hline $\begin{array}{l}\left.L_{x} \times L_{y} \text { (units of } d_{i 0}\right) \\
\Delta / d_{i 0}\end{array}$ & $\begin{array}{l}200 \times 30 \\
0.5\end{array}$ & & & \\
$M_{i} / m_{e}$ & 256 & & & \\
$v_{e} / c$ & 0.017 & $T_{e}=0.15 \mathrm{keV}$ & $T_{e}=1 \mathrm{keV}$ & $c / v_{A 0}=273$ \\
$T_{i} / T_{e}$ & 5 & $T_{i}=0.73 \mathrm{keV}$ & $T_{i}=5 \mathrm{keV}$ & $\begin{array}{l}n_{0}=0.34 \mathrm{~cm}^{-3} \\
d_{i 0}=400 \mathrm{~km} \\
\text { for } B_{0}=30 \mathrm{nT} \\
n_{b} / n_{0}\end{array}$ \\
$B_{g} / B_{0}$ & 0.1 & & & $c / v_{A b}=86$ \\
\hline
\end{tabular}

Table 4 Simulation $\mathrm{C}$, both electron and ion masses physical

\begin{tabular}{llll}
\hline $\begin{array}{l}\text { Input params } \\
\text { Simulation C }\end{array}$ & Value & $\begin{array}{l}\text { Both masses } \\
\text { are physical }\end{array}$ & $\begin{array}{l}\text { Derived from } \\
\text { input params }\end{array}$ \\
\hline $\begin{array}{l}\left.L_{x} \times L_{y} \text { (units of } d_{i 0}\right) \\
\Delta / d_{i 0}\end{array}$ & $40 \times 20$ & & \\
$M_{i} / m_{e}$ & 0.5 & & \\
$v_{e} / c$ & 1836 & $T_{e}=1 \mathrm{keV}$ & $c / v_{A 0}=273$ \\
$T_{i} / T_{e}$ & 0.045 & $T_{i}=5 \mathrm{keV}$ & $\begin{array}{l}n_{0}=0.34 \mathrm{~cm}^{-3} \\
d_{i 0}=400 \mathrm{~km} \\
\text { for } B_{0}=30 \mathrm{nT} \\
n_{b} / n_{0}\end{array}$ \\
$B_{g} / B_{0}$ & 5 & & $c / v_{A b}=86$ \\
\hline
\end{tabular}

Simulation A is more realistic for electron scale phenomena than for ion scale processes. Simulation B, described in Table 3, differs only in the input electron thermal velocity but is more realistic for ions in terms of the resulting electron and ion temperatures.

Simulations $\mathrm{C}$ with the physical mass ratio, $M_{i} / m_{e}=1836$ also have been performed with a smaller simulation box and for shorter times than in simulations A and B (Table 4).

The boundary conditions for these simulations are conducting in $y$ and periodic in $x$. An initial perturbation in the magnetic field is introduced in the neighborhood of $\left(x_{0}=100 d_{i 0}\right.$, $y_{0}=15 d_{i 0}$ ) to speed up reconnection and create an $x$-line at that point. Unlike the global perturbation of the GEM Challenge (Eq. (5a)-(5c)) of Birn et al. (2001), our simulations are initialized by a local perturbation of the out-of-plane vector potential $A_{z}$ that takes the form of a Gaussian of half-width equal to the half-width of the Harris current sheet $\left(0.5 d_{i 0}\right)$. The amplitude of the perturbation is chosen so that the maximal vertical magnetic fields are $B_{y} \approx \mp 0.35 B_{0}$ located at $\left(x-x_{0}, y-y_{0}\right) \approx\left(\mp 0.35 d_{i 0}, 0\right)$. Although weak spontaneous tearing of the Harris sheet away from the imposed perturbation occurs late in the simulation, it does not significantly affect any of the results presented in this study.

In Fig. 1 the reconnection geometry in the reconnection plane, $(x, y)$ is shown at time $t \Omega_{i 0} \approx 30$ in Simulation B. For $B_{0}=30 \mathrm{nT}$ (Cattell et al. 2005), and the physical ion mass, this is a physical time of $\approx 11$ secs. The compression in $B_{y}$ field lines corresponding to the magnetic flux pile-up front is evident in Fig. 1a. Figure 1a also displays the out-ofplane reconnection electric field, $E_{z}$, which pervades both the inflow and outflow regions, but peaks at the moving front, where there is a large curl of $\mathbf{B}$ due to pileup and where 
Fig. 1 Front characteristics from simulation $B$ at $t=30.4 \Omega_{i}^{-1}$. Front is to right of vertical dashed line, $\Delta x= \pm 23 d_{\text {io }}$ from $x$-line $\left(x=100 d_{i 0}\right)$. (a) Out-of-plane "reconnection" field, $E_{z}$ in dimensionless units, $\left(E_{z} / B_{0}\right)\left(c / v_{A 0}\right)$ with in-plane $B$-field lines and $B_{y}$ pileup front. (b) Log of ion density over initial background density shows density front. (c) Log of ion pressure over initial background pressure shows pressure front
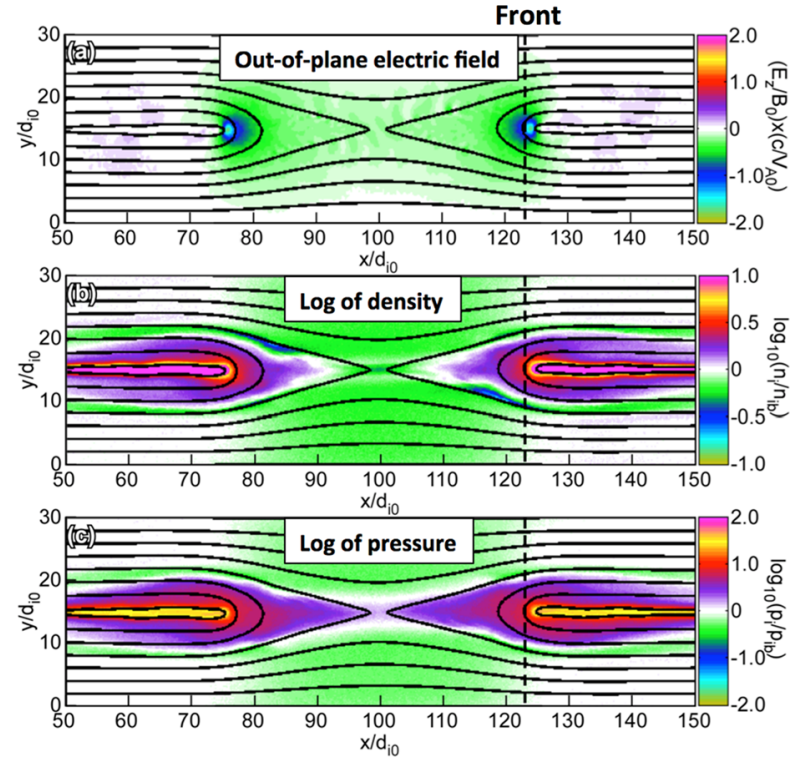

work is performed on ions by $E_{z}$ (to be shown later). In the inflow regions $E_{z}$ pushes flux tubes towards the separatrix. The reconnection field, $E_{z}$, at the $x$-point is a measure in 2D simulations of the increase in reconnected magnetic flux piercing the neutral line between the $x$-point and one edge of the simulation box. The dimensionless reconnection rate in this sense is given by $\left(c / v_{A}\right) \cdot E_{z}\left(x\right.$-pt.) $/ B_{0}$, where $v_{A}$ is the Alfven speed in the inflow plasma upstream of the dissipation region. Numerically this dimensionless rate is here on the order of 0.1, in agreement with other simulation results (e.g., Shay et al. 2001). The vertical striations in $E_{z}$ visible near the $x$-point in Fig. 1a come from electromagnetic whistler waves which permeate the inflow region and modulate the reconnection rate (Goldman et al. 2014). These waves are generated by Čerenkov emission from fast electron phase space holes.

In Fig. $1 b$, the log of the ion density divided by the background density, $n_{b}$, is shown. The original Harris sheet (cross-field current sheet) has torn, and an $x$-point has formed at the center of the simulation box with reconnected field lines pulling away from it on either side due to magnetic tension. Both the inflow and outflow-exhaust of particles originate from the initial low-density uniform background plasma (lobe or plasmasheet). Inflowing particles are less dense than $n_{b}$, becoming more dense towards the pile-up region in the outflow. The pressure difference between the low and high density regions plotted in Fig. 1c opposes the magnetic tension of reconnected field lines and leads to a moving front in both pressure and magnetic field near the compressed remaining Harris sheet.

Once the ambient (Harris) current sheet has torn and "unzipped" on both sides of the $x$-point a new (replacement) current sheet forms in the out-of-plane (z) direction. In Fig. 2 the $z$-components of the electron and ion currents are displayed. The new electron current dominates the new ion current sheet at the separatrix near the $x$-point, but the ion current begins to dominate near the center of the low-density side of the front and is highly dominant near the pile-up front, around $x / d_{i 0}=100 \pm 23$ (not shown). The reconnection field, $E_{z}$ performs significant work on the ion current, $J_{i z}$ in the region where both are large, near the front. This will be treated later. 

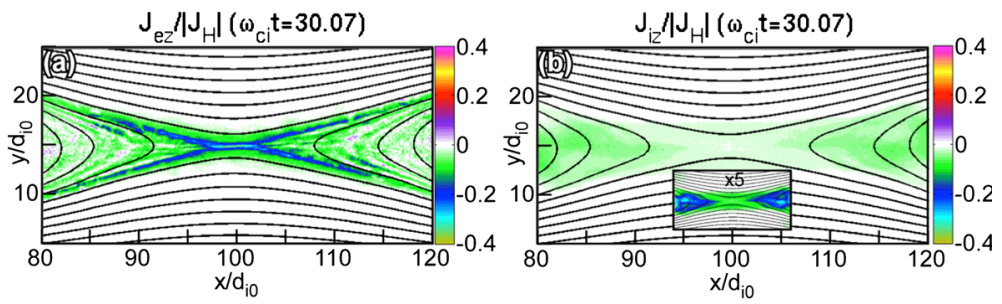

Fig. 2 Replacement current sheet after reconnection. From Simulation A: (a) shows electron out-of-plane current, $J_{\mathrm{ez}}$ at $\Omega_{c i} t=30.1$, in units of peak Harris-sheet diamagnetic current; (b) shows smaller ion out-of-plane current, $J_{\mathrm{iz}}$ in same units (inset: same region with magnitude multiplied by a factor of 5). Both aspect ratios are correct

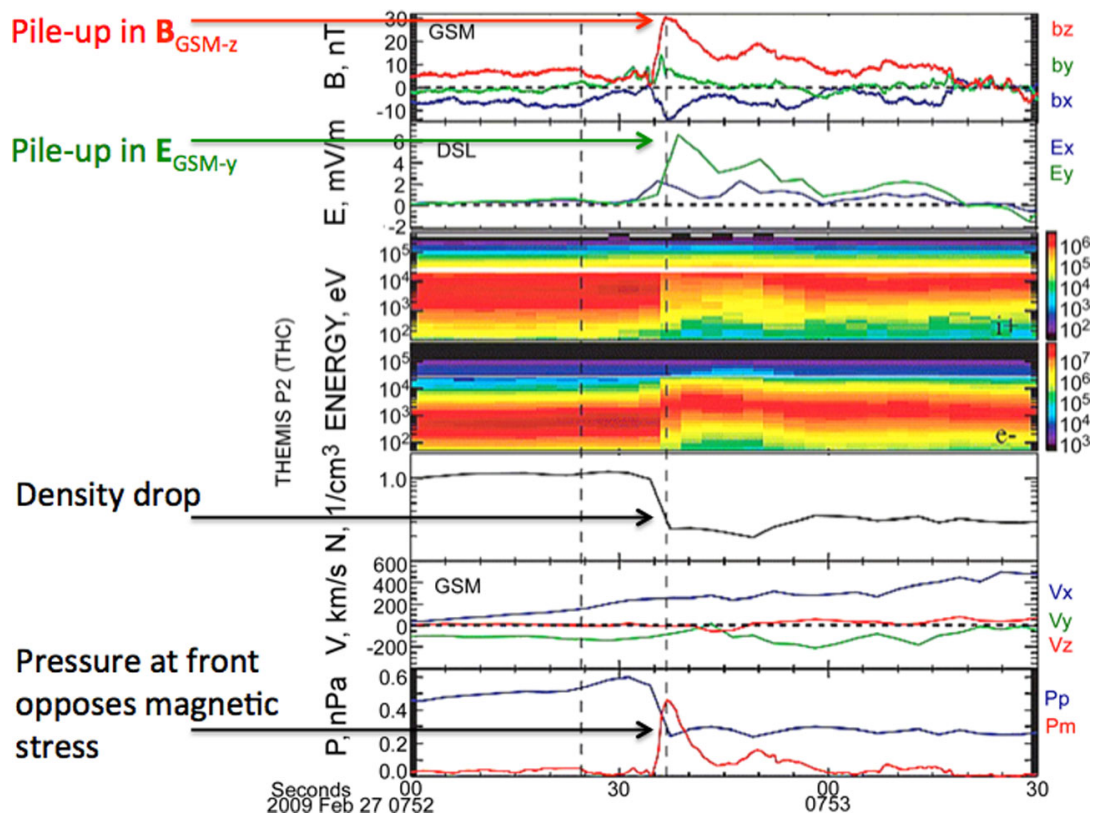

Fig. 3 Data from Themis observation of a dipolarization front in the magnetail, Feb. 27, 2009 (Runov et al. 2009). Note, $x$-point is to right. Arrows show features which are also found in simulations (see Fig. 4)

\subsection{Dipolarization Measurements in the Tail Compared with Simulation}

The dynamic features of tail reconnection described in Fig. 1 are confirmed in measurements of dipolarization events in Earth's magnetotail on Feb. 27, 2009 (Runov et al. 2009). In Fig. 3 the results of Themis measurements are shown for an especially well-behaved dipolarization front (DF) event which is noteworthy because it does not exhibit significant "flapping" in the $\pm z$-GSM direction. The time-interval of the data is less than 90 seconds.

The pileup in $B_{\mathrm{GSM}-z}$ in Fig. 3 corresponds to the pileup in $B_{y}$ seen in the simulation in Fig. $1 \mathrm{~b}$, the peak in $E_{\mathrm{GSM}-y}$ in Fig. 3 corresponds to the peak in $E_{\text {recon }}=E_{z}$ in the simulation in Fig. 1b and the density drop in Fig. 3 corresponds to the drop of density at the pressure front in the simulation shown in Fig. 1a. 
Fig. 4 Line plots from

Simulation A as functions of $x$ at $y=15$ : (a) Comparison of magnetic and ion pressures across pile-up front. (b) Piled-up field, $B_{y}(x) / B_{0}$, negative of (magnified) reconnection field, $-\left(E_{z}(x) / B_{0}\right) \cdot\left(c / v_{A 0}\right)$, and drift, $-\left(E_{z} B_{y} /|B|^{2}\right) \cdot\left(c / v_{A 0}\right)$ (a measure of front velocity)

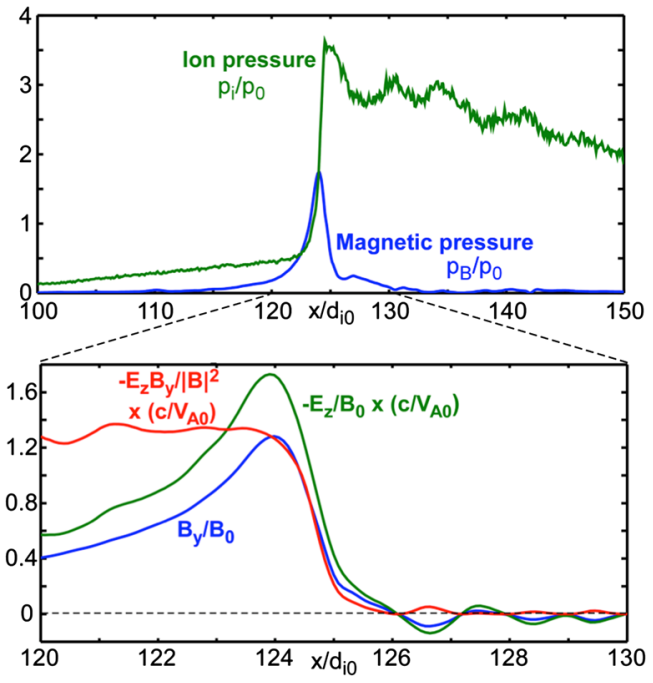

Similarities and differences between Themis observations and simulation results on either side of the front are compared below for Themis and simulation data:

- The width of the peak DF is a few $d_{i 0}$ for both simulation and Themis data.

- The Themis drop in density across the DF is roughly by a factor of five (from $n=1 \mathrm{~cm}^{-3}$ to $n=0.2 \mathrm{~cm}^{-3}$ ), whereas according to Fig. 1a the simulation density drop is about a factor of seven (from $n=20 n_{b}$ to $n=3 n_{b}$ ). However the simulation density drop is partially determined by the assumed ratio of Harris density to background density, $n_{o} / n_{b}$ which was taken as equal to 10 , so this agreement could be accidental.

- The ion pressure measured by Themis drops by a factor of two from the high density side to the low density side (from $p=0.6$ to $p=0.3 \mathrm{~Pa}$ ) in Fig. 3. This is half as large as the measured density drop, consistent with ion heating on the low density side. The pressure drop across the DF (from left to right in Fig. 3) is larger than the magnetic pressure drop.

- The line plots in Fig. 4 show that the results of Simulation $A$ are similar to the Themis measurements. At the simulation time $\Omega_{i 0} t=30$ depicted, the front has moved out to a distance $\approx 24 d_{i 0}$ from the $x$-point. The distance from the $x$-pt of the DF observed by Themis is unknown but probably much larger-on the order of $10 R_{E}$. Figure 4 a shows a drop in both magnetic and ion pressure in the simulation across the DF towards the $x$-point (towards the low-density side); the magnetic pressure drop is larger in the simulation than in the Themis data. Figure $4 \mathrm{~b}$ consists of line-plots across the front of the pile-up field, $B_{y}(x) / B_{0}$, the reconnection field, $\left(E_{z} / B_{0}\right) \cdot\left(c / v_{A 0}\right)$, and the drift velocity, $-c E_{z} B_{y} /\left(v_{A 0}\right.$. $\left.|B|^{2}\right)$, which is a measure of the front-velocity, $v_{F}$. On the upstream side of the front (towards the $x$-point), $v_{F} \approx 1.3 v_{A 0}$.

- The components of the ion flow velocity, $\mathbf{u}$, are shown in Fig. 5. The outflow velocity (jet), $u_{i x}$, is on the order of $v_{A 0}$ upstream and $2 v_{A 0}$ downstream. Hence, $u_{i x}$ upstream is moving slightly slower than the front, although in certain other simulations it is faster (Liu et al. 2012). In the Themis data, $u_{i x}$ increases across the front in the upstream direction unlike in the simulation, where $u_{i x}$ is fairly flat on the $x$-pt. side of the front (upstream), but increases across the front and downstream, where it plateaus at $2 v_{A 0}$ just below the 

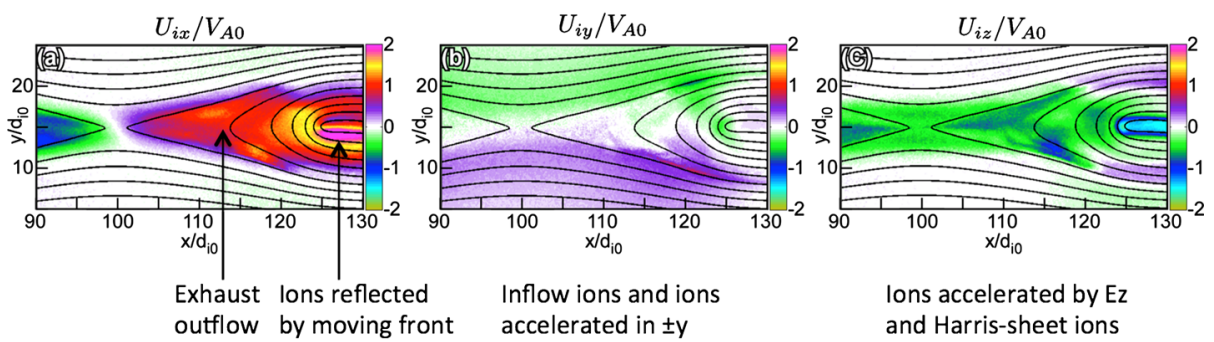

Ions accelerated by Ez and Harris-sheet ions

Fig. 5 Components of ion flow velocity, $\mathbf{u}_{i}$ (velocity moment of ion distribution function) in units of Harris Alfven velocity: (a) $u_{i x}$ : ions upstream of front (at $x=123 d_{i 0}, y=15 d_{i 0}$ ) move at $u_{i x} \approx v_{A 0}$, while reflected ions downstream of front (at $x=125-130 d_{i 0}, y=14 d_{i 0}$ ) move at $u_{i x} \approx 2 v_{A 0}$. (b) $u_{i y}$ : $\pm y$ inflow ions and ions accelerated in $\pm y$ by the Hall electric field just inside the separatrices closer to the $x$-point or by $B_{y}$ closer to the front. (c) $u_{i z}$ : ions accelerated out-of-plane by reconnection field, $E_{z}$, and diamagnetic ions in upstream untorn Harris current sheet with $u_{i z} \approx 1.5 v_{A 0}$

symmetry axis (due to the small guide field). A possible explanation for the faster jet is that it is due to downstream ions which are reflected by the pile-up field, $B_{y}$, in the frame of the front (Drake et al. 2014; Eastwood et al. 2015). The ion flow component, $u_{i y}$, upstream of the front is largest in the inflow (outside the separatrix) and near reconnected field-lines inside the separatrix. Just inside the separatrix and sufficiently upstream of the front they are accelerated in $\pm y$ by the Hall electric field (discussed in next section). Closer to the front, $u_{i y}$ is determined by the combination of the reconnection electric field, $E_{z}$ and the local Lorentz forces. Finally, the component, $u_{i z}$, is due to $E_{z}$ upstream of the front. Downstream it becomes the diamagnetic drift associated with the as-yetunreconnected ambient current sheet.

- The ambient current sheet is much thicker in the Themis observations than the current sheets used in most simulations. As a result the opening angle of the exhaust in thin current-sheet simulations is large enough for low density outflow to sandwich the high density unreconnected thin current sheet. Near the DF the thickness of this unreconnected high-density current sheet appears to have roughly doubled.However, this simulation result is still very different from the physical Themis observations which show that the entire DF butts up against the high density unreconnected cross-tail current sheet which can be as thick as 20 local ion inertial lengths (J. Eastwood, H. Hietel, private communication). A thicker current sheet downstream of the DF could be associated with a larger region in $\pm z$-GSM of $B_{z-G S M}$, and more effective braking of the DF relative to the ion outflow behind it.

\subsection{Useful Coordinate Systems for Describing Simulations}

In order to visualize and discuss the magnetic and electric fields, electron and ion flows and diffusion regions found in our reconnection simulations we define in Fig. 6 two threedimensional rectilinear coordinate systems bases for the simulations in addition to the Cartesian system, $\{x, y, z\}$ : a B-field-aligned basis, $\left\{\hat{\mathbf{e}}_{\|}, \hat{\mathbf{e}}_{\perp 1}, \hat{\mathbf{e}}_{\perp 2}\right\}$ and an in-plane-B-componentaligned basis, $\left\{\hat{\mathbf{e}}_{=}, \hat{\mathbf{e}}_{z}, \hat{\mathbf{e}}_{\perp 1}\right\}$.

The B-field-aligned basis vectors, $\left\{\hat{\mathbf{e}}_{\|}, \hat{\mathbf{e}}_{\perp 1}, \hat{\mathbf{e}}_{\perp 2}\right\}$, are defined as follows:

- $\hat{\mathbf{e}}_{\|}$is parallel to the total field, B. This is the direction of the bipolar electrostatic fields associated with electron phase-space holes to be discussed below. 


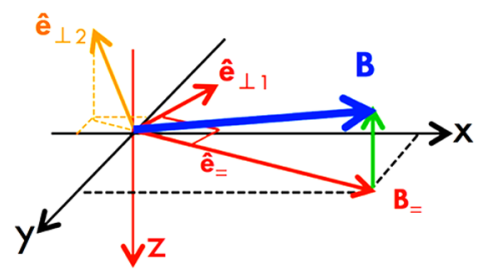

Fig. 6 Field-aligned coordinates and in-plane field-aligned coordinates defined. The blue vector, $\mathbf{B}$, is the total local magnetic field. The green vector in the $-z$ direction is the combined Hall and guide field, $\mathbf{B}_{H}+\mathbf{B}_{g}$. The red vector, $\mathbf{B}_{=}$is the in-plane projection of $\mathbf{B}$. The unit vectors associated with different Cartesian coordinate systems are explained in the text

- $\hat{\mathbf{e}}_{\perp 1}$ is in the $\mathbf{B} \times \hat{\mathbf{e}}_{z}$ direction, which is in the reconnection $x-y$ plane, perpendicular to in-plane magnetic field lines. It is nearly parallel to $\pm y$ ( $\pm z-\mathrm{GSM})$ for small exhaust opening angles.

$\circ \hat{\mathbf{e}}_{\perp 2}$ is in the $\mathbf{B} \times \hat{\mathbf{e}}_{\perp 1}=-\hat{\mathbf{e}}_{z} B^{2}+\mathbf{B}\left(\hat{\mathbf{e}}_{z} \cdot \mathbf{B}\right)$ direction, which is the $-z$-direction when $\mathbf{B}$ has a negligible $z$-component.

The in-plane-B-component-aligned basis vectors, $\left\{\hat{\mathbf{e}}_{=}, \hat{\mathbf{e}}_{z}, \hat{\mathbf{e}}_{\perp 1}\right\}$ are useful for describing Hall fields and currents. They are defined as follows

○ $\hat{\mathbf{e}}_{=}$is the direction parallel to the vector component of $\mathbf{B}$ in the $x-y$ plane. It is mainly in the $x$-direction.

$\circ \mathbf{e}_{z}$ is in the $z$-direction.

- $\hat{\mathbf{e}}_{\perp 1}$ is again in the $\mathbf{B} \times \hat{\mathbf{e}}_{z}$ direction as in the field-aligned coordinate system above. It is perpendicular to $\hat{\mathbf{e}}_{=}$.

\section{$1.5 \mathrm{E}(x, y)$ Components in Different Coordinate Systems}

In Fig. 7 the components of the total local electric field, $\mathbf{E}(x, y)$, found from Simulation A at time 30 are displayed using both $\left\{\hat{\mathbf{e}}_{x}, \hat{\mathbf{e}}_{y}, \hat{\mathbf{e}}_{z}\right\}$ basis vectors and $\mathbf{B}$-field-aligned basis vectors, $\left\{\hat{\mathbf{e}}_{\|}, \hat{\mathbf{e}}_{\perp 1}, \hat{\mathbf{e}}_{\perp 2}\right\}$.

The in-plane component, $E_{\perp 1}$ in Fig. 7e peaks near the separatrices and points inwards, towards the $y=15$ symmetry axis (as is more evident in $E_{y}$ ). It is mainly electrostatic (to be elucidated later in terms of Hall physics). This field-component accelerates ions entering the exhaust across the separatrices, upstream of the front.

The component, $E_{\|}$, parallel to the magnetic field is also electrostatic and has bipolar structure near the separatrices. This is especially evident on the upper right separatrix, from around 15 to $25 d_{i 0}$ from the $x$-pt. This bipolar $E_{\|}$corresponds to moving phase space hole structures. There are also weak patchy spots of $E_{\|}$with both signs in the central exhaust (note magnification of $E_{\|}$by a factor of five in this region).

The out-of-plane component $E_{z}$ or $E_{\perp 2}$ is the reconnection electric field, which is most intense near the pile-up front, as already discussed in connection with Fig. 1 . At the $x$-point and in the nearby central exhaust $E_{z}$ and $E_{\perp 2}$ are much weaker but still dominate the other electric field components.

\subsection{Simulation $\mathrm{E}$ and $\mathrm{B}$ Fields in $\mathrm{mV} / \mathrm{m}$ and $\mathrm{nT}$ Compared with Magnetotail Measurements}

In this section we describe how to interpret the magnitude of simulation $\mathbf{E}$ and $\mathbf{B}$-fields found in simulations in terms of physical units. Electric and magnetic fields in simulations both 

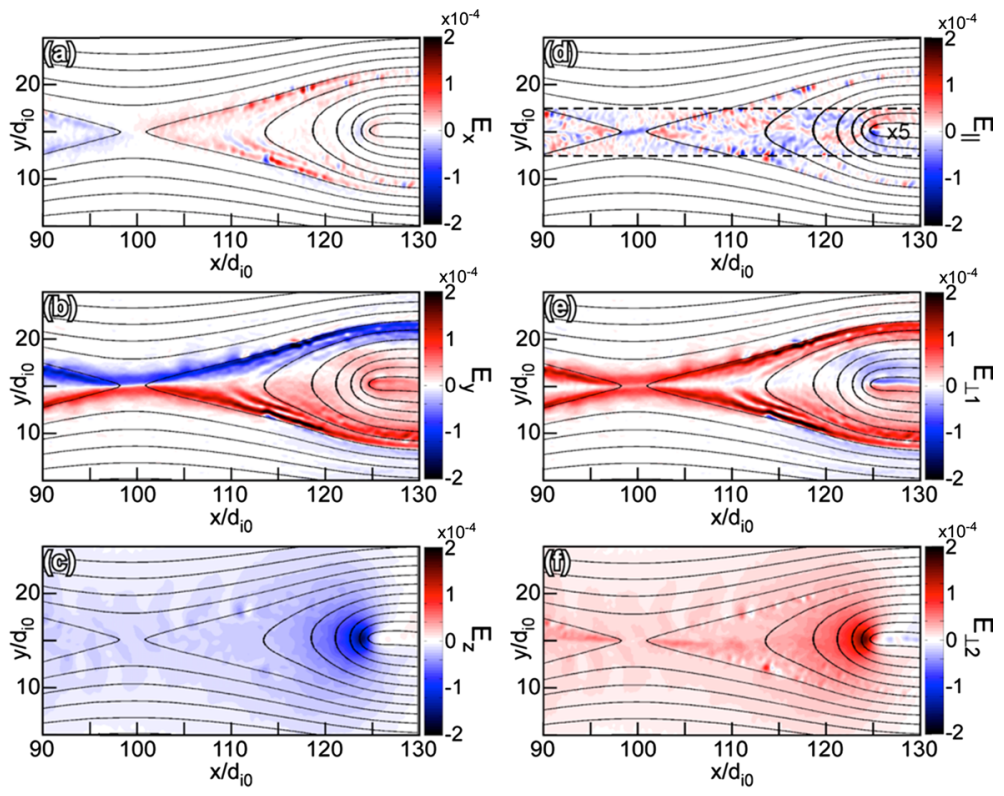

Fig. 7 Components of $\mathbf{E}(x, y)$ in simulation coordinates (a)-(c) and in field-aligned coordinates (d)-(f). In the region between the dashed lines in (d), $E_{\|}$is magnified by a factor of 5

have the same dimensionless units. They are both scaled by $\sqrt{4 \pi n_{0} M_{i} c^{2}}=\left(c / v_{A 0}\right) \cdot B_{0}$. As in Eq. (1a), (1b), $c / v_{A 0}$ is the ratio of the speed of light to the Harris ion Alfven speed given in Tables 2, 3, and 4 for Simulations A, B and C.

1. To convert a simulation magnetic field, $B_{\text {sim }}$ into nanoTesla, simply use an appropriate measured value of $B_{0}$ (in nT) and multiply by $c / v_{A 0}$.

Examples From Fig. 4, the peak pile-up magnetic field, $B_{y}$, is $1.3 \times 10^{-2}$. Since $c / v_{A 0}=100$ in this simulation (Simulation A), $B_{y}=1.3 B_{0}$, with $B_{0}$ in nT. Hence, the pile-up $B_{y}$ is on the order of the asymptotic reconnection $B$-field, $B_{0}$. From the Themis tail measurements depicted in Fig. 3, the measured pile-up field $B_{\mathrm{GSM}-z}$ is on the order of $30 \mathrm{nT}$, while the local $B_{x}$ has a peak magnitude of $10 \mathrm{nT}$. The Themis spacecraft are not located in the asymptotic inflow region where $B_{0}$ can be measured.

The magnitude of the measured Hall magnetic field, $B_{\mathrm{GSM}-y}$ can also be compared to simulation. In Simulation C (with physical mass ratio) the peak Hall magnetic field in simulation units is $B_{\mathrm{H}-\mathrm{sim}}=1.2 \times 10^{-3}$ and $c / v_{A 0}=275$. For Cattell's data (Cattell et al. 2005) $B_{0}=30 \mathrm{nT}$, so $B_{H}=1.2 \times 10^{3} \cdot 275 \cdot 30=10 \mathrm{nT} \approx B_{0} / 3$, which is a typical tail Hall magnetic field.

2. To convert a simulation electric field, $E_{\text {sim }}$ into $\mathrm{mV} / \mathrm{m}$ first convert $B_{0}$ into gaussian field units and then convert to the SI unit of $\mathrm{V} / \mathrm{m}$. This is accomplished by multiplying $B_{0}$ in Tesla by $\left(c / v_{A 0}\right)$ and then by $c=3 \times 10^{8} \mathrm{~m} / \mathrm{s}$.

Examples From Fig. 7 (Simulation A), with $E_{\perp 1} \approx 10^{-4}$ and $c / v_{A 0}=100$, and $B_{0}=30 \mathrm{nT}$, $E_{\perp 1} \approx 10^{-4} \cdot 100 \cdot\left[\left(3 \times 10^{8}\right) \cdot\left(30 \times 10^{-9}\right)\right]=0.09 \mathrm{~V} / \mathrm{m}=90 \mathrm{mV} / \mathrm{m}$. From the more realistic Simulation $C$, with physical mass ratio, the peak $E_{\perp}$ is $E_{\perp 1}=3 \times 10^{-5}$, so, $E_{\perp 1}=$ 
$3 \times 10^{-5} \cdot 275 \cdot\left[\left(3 \times 10^{8}\right) \cdot\left(30 \times 10^{-9}\right)\right]=0.075 \mathrm{~V} / \mathrm{m}=75 \mathrm{mV} / \mathrm{m}$. Both values are close to peak values measured in the tail (Wygant et al. 2005).

3. To get a Poynting flux in $\mathrm{W} / \mathrm{m}^{2}$ use the SI formula with $\mu_{0}=4 \pi \times 10^{-7}$ :

$$
\mathbf{S}_{\mathrm{SI}}=\frac{\mathbf{E} \times \mathbf{B}}{\mu_{0}} .
$$

Example From Simulation C, $S_{\text {Hall }}=\left(75 \times 10^{-3}\right) \cdot\left(10 \times 10^{-9}\right) /\left(4 \pi \times 10^{-7}\right)=0.6 \mathrm{~mW} / \mathrm{m}^{2}$.

\subsection{Summary of Geometry of Tail Reconnection}

The regions of interest in tail reconnection physics described in this section and to be described in the remainder of this Chapter are as follows:

(A) Inflow region

(B) $x$-point and magnetic separatrix neighborhoods (may be several if there is secondary reconnection)

(C) Entire exhaust region from $x$-point to pile-up front

(a) Electron diffusion region around $x$-point where magnetic field lines reconnect

(b) Ion diffusion region

(c) Frozen-in ion region

(D) Pile-up front regions on either side of an $x$-point (Earthward and anti-Earthward)

(E) Region of compressed un-torn cross-field current sheet in front of pile-up region

A central goal of MMS is to probe "diffusion regions," yet the meaning and location of "diffusion regions" still remain unclear and are often ambiguous. In the next sections we explain a number of complementary descriptions of diffusion regions, the relation between them, and the results of two-dimensional PIC simulations for a number of suggested signatures of diffusion region in widely-separated regions ( $\mathrm{B}, \mathrm{C}$ and $\mathrm{D}$, above) of the magnetotail reconnection geometry.

\section{Diffusion Regions and Hall Physics}

\subsection{What Is Meant by Diffusion Region?}

Ideal fluid models such as MHD do not permit reconnection because magnetic field lines are frozen-into the fluid, magnetic flux is conserved and the topology of magnetic field connectivity is preserved. These constraints are all removed when collisional resistivity is added to the MHD equations. In magnetospheric reconnection, collisions are completely negligible. However, the constraints of ideal MHD are also removed when MHD is replaced with collisionless kinetic theory, as expressed in Particle-In-Cell (PIC) simulations. Regions in PIC simulations where any of the above constraints imposed by ideal MHD are broken are of physical interest. Those nonideal regions and still others have all come to be called diffusion regions (Vasyliunas 1975, 1972) or dissipation regions, even when there is no resistive diffusion of magnetic field lines and, strictly speaking, no dissipation.

Violation of any of the constraints such as frozen-in magnetic field lines or magnetic flux conservation in a region does not imply that magnetic reconnection (topology change) occurs in or near that region (Newcomb 1958; Hornig and Schindler 1996; 
Biskamp 2000). Most or all presently-employed measures of diffusion regions in 2D-PIC simulations are necessary but not sufficient indicators of regions in which reconnection occurs. Since a principal purpose of MMS is to probe diffusion regions associated with magnetopause and magnetotail reconnection it is essential to clarify the various (often different) meanings of diffusion region.

A number of common measures of diffusion regions are based on (collisionless) "generalized Ohm's Laws," usually obtained from a fluid momentum equation for either species (or for an MHD single fluid). In PIC simulations the fluid momentum equation should be regarded as an exact consequence of taking a first velocity moment of the collisionless Boltzmann equation. No equation of state closure model needs to be assumed since each of the terms in the generalized Ohm's Law can be evaluated numerically from the simulation.

A major advance in understanding the limitations of MHD treatments of reconnection occurred when kinetic PIC simulations showed that the Hall term in the generalized Ohm's Law leads to separate diffusion regions for electrons and for ions.

\subsection{Conditions for a Magnetofluid to Be Ideal}

In an ideal ion or electron magneto-fluid, magnetic lines of force are frozen-in and move with fluid elements. Whether a magnetofluid can be considered ideal or nonideal in a spatial region can be determined from fluid momentum equations for the ion or electron fluid velocity field, $\mathbf{u}(\mathbf{r}, t)$. The fluid momentum equation for either electrons or ions may be written as one vector equation (2a) or decomposed into a parallel (1D) part and perpendicular (2D) parts, (Eqs. (2b), (2c)), by using B-field-aligned basis vectors:

$$
\begin{aligned}
& \mathbf{E}+\frac{\mathbf{u}}{c} \times \mathbf{B}=\mathbf{R} \\
& \mathbf{E}_{\|}=\mathbf{R}_{\|}, \\
& \mathbf{E}_{\perp}+\frac{\mathbf{u}_{\perp}}{c} \times \mathbf{B}=\mathbf{R}_{\perp}
\end{aligned}
$$

For example if $\mathbf{u}(\mathbf{r}, t)$ in Eq. (2a) is the electron fluid velocity, then $\mathbf{R}$ would consist of an electron inertial term and an exact electron pressure term. In spatial regions where all three components of $\mathbf{R}(x, y)$ are essentially zero, the fluid is said to be ideal - that is, well-defined moving magnetic field lines are frozen-into the perpendicular motion of fluid elements and magnetic flux is conserved. Although $\mathbf{R}=0$ in a region is a sufficient condition for the fluid to be ideal in that region it is not a necessary condition. (Note, the speed of light, $c$, in Eqs. (2a)-(2i) will sometimes be taken as $c=1$ in later equations.)

In spacecraft measurements of reconnection events, both $\mathbf{E}_{\|}$and (therefore) its curl (in Eqs. (2e) and (2f), below) are often assumed to be negligibly small in a region, so a sufficient condition for a fluid species to be ideal in that region is $\mathbf{R}_{\perp}=0$. Simulations afford an opportunity to examine parallel electric fields. In regions where $\mathbf{E}_{\|}$(and its curl) are not negligible, the condition $\mathbf{R}_{\perp}=0$ is not a sufficient condition for idealness, since it no longer guarantees that the motion of lines of $\mathbf{B}$ are frozen into the perpendicular flows, $\mathbf{u}_{\perp}$. Instead, the condition $\mathbf{R}_{\perp}=0$ only implies that the species cross-field fluid velocity, $\mathbf{u}_{\perp}$, is equal to the $\mathbf{E} \times \mathbf{B} / B^{2}$ particle drift velocity:

$$
\mathbf{R}_{\perp}=0 \quad \Rightarrow \quad \mathbf{u}_{\perp}=\frac{\mathbf{E} \times \mathbf{B}}{B^{2}}=\text { drift. }
$$

However the drift velocity, $\mathbf{E} \times \mathbf{B} / B^{2}$, may or may not be the velocity of moving $B$-field lines. The problem is that in order for moving magnetic field lines to be well-defined (i.e., to 
be "conserved," "preserved" or, for moving fluid elements,to be tied to magnetic field lines) there is an additional condition on the parallel electric fields (Newcomb 1958; Vasyliunas 1972; Hesse and Schindler 1988; Schindler et al. 1991):

$$
\begin{aligned}
& \mathbf{R}_{\perp}=0 \quad \text { and } \quad \mathbf{B} \times\left(\nabla \times \mathbf{E}_{\|}\right)=0 \\
& \Rightarrow \quad \text { conserved moving magnetic field lines (frozen-in) }
\end{aligned}
$$

In regions where $\mathbf{B} \neq 0$ the condition $\mathbf{B} \times\left(\nabla \times \mathbf{E}_{\|}\right)=0$ in Eq. (2e) is equivalent to $\left(\nabla \times \mathbf{E}_{\|}\right)_{\perp 1,2}=0$. (Note that magnetic null points, at which all three components of $\mathbf{B}$ vanish automatically satisfy $\mathbf{B} \times\left(\nabla \times \mathbf{E}_{\|}\right)=0$. However in the present $2 \mathrm{D}$ simulations of component reconnection, with guide field $\mathbf{B}_{g}=0.1 \mathbf{B}_{0}$, there are no magnetic null points.)

Equations (2e) do not guarantee that magnetic flux is conserved. For $f l u x$ to be conserved when $\mathbf{R}_{\perp}=0$ not only must $\left(\nabla \times \mathbf{E}_{\|}\right)_{\perp 1,2}=0$, but also $\left(\nabla \times \mathbf{E}_{\|}\right)_{\|}$must be zero. That is, all three components of $\nabla \times \mathbf{E}_{\|}$must vanish:

$$
\begin{aligned}
& \mathbf{R}_{\perp}=0 \quad \text { and } \quad \nabla \times \mathbf{E}_{\|}=0 \\
& \Rightarrow \quad \text { conservation of magnetic flux and field lines }
\end{aligned}
$$

Equations (2e) and (2f) are sufficient but not necessary conditions for idealness. The necessary conditions do not require that $\mathbf{R}_{\perp}=0$ but rather that $\nabla \times \mathbf{R}_{\perp}=0$. Since $\mathbf{R}_{\perp}$ is defined as $\mathbf{R}_{\perp}=\mathbf{E}_{\perp}+\mathbf{u} \times \mathbf{B}$, the necessary and sufficient conditions for an ideal magnetoplasma are (Hesse and Schindler 1988; Schindler et al. 1991).

$$
\begin{aligned}
\mathbf{B} & \times \nabla \times(\mathbf{E}+\mathbf{u} \times \mathbf{B})=0 \\
\quad & \Rightarrow \text { conserved moving magnetic field lines (frozen-in) } \\
\nabla & \times(\mathbf{E}+\mathbf{u} \times \mathbf{B})=0 \Rightarrow \text { conservation of magnetic flux and field lines }
\end{aligned}
$$

Note that the flux conservation condition, Eq. (2h) guarantees frozen-in magnetic field lines (Eq. ( $2 \mathrm{~g}))$ because all three components of $\nabla \times(\mathbf{E}+\mathbf{u} \times \mathbf{B})=0$ must vanish, so the components perpendicular to $\mathbf{B}$ (as well as parallel) are zero, as required by Eq. (2g). However, frozen-in field lines (Eq. (2g)) do not guarantee flux conservation (Eq. (2h)) since Eq. (2g) does not require that the component of Eq. (2h) parallel to $\mathbf{B}$ must vanish. Both Eqs. (2g) and (2h) are automatically satisfied in a spatial region where $\mathbf{E}+\mathbf{u} \times \mathbf{B}=0$, but may be satisfied even in regions where $\mathbf{E}+\mathbf{u} \times \mathbf{B} \neq 0$. For example, Eqs. ( $2 \mathrm{~g})$ and (2h) are both satisfied if $\mathbf{E}+\mathbf{u} \times \mathbf{B}=\nabla \psi$, where $\psi$ is a scalar function, since the curl of a gradient vanishes (Vasyliunas 1972; Hornig and Schindler 1996). Under these conditions the flow velocity of the species in question, $\mathbf{u}_{\perp}$, is not simply the $\mathbf{E} \times \mathbf{B}$ drift, $\mathbf{v}_{D}$, yet the field lines are still frozen-in-moving with the fluid elements at $\mathbf{u}_{\perp}$.

Assuming that the electric field, E, in Eq. (2h) is not entirely electrostatic, Faraday's law allows the flux conservation condition to be expressed as

$$
\partial_{t} \mathbf{B}-\nabla \times\left(\mathbf{u}_{s} \times \mathbf{B}\right)=-\nabla \times \mathbf{R}_{s}=0
$$

Here, $\boldsymbol{\nabla} \times\left(\mathbf{u}_{s} \times \mathbf{B}\right)$ is the usual convective term. For an ideal electron or ion fluid $(s=e, i)$, the equation $\partial_{t} \mathbf{B}-\nabla \times\left(\mathbf{u}_{s} \times \mathbf{B}\right)=0$ implies flux conservation $\partial_{t} \Phi=0$ with respect to the fluid motions of either electrons or ions (or of a single MHD-fluid) when integrated over a flux surface whose boundary is moving with the fluid. When Eqs. (2h), (2i) are violated for either species so that magnetic flux is not conserved, the flow is not line-preserving 
and magnetic field lines are not frozen-in to the cross-field fluid motion of that species (Newcomb 1958). Violation of Eqs. (2g), (2h), (2i) will be studied later. Hall physics for the generalized Ohm's law of each species will be treated first. Hall physics allows $\mathbf{B}$ to be frozen-in to the electron fluid in regions where it is not frozen-into the ion fluid.

\subsection{Hall Physics and Hall Fields}

We employ the following forms of generalized Ohm's Law for each species:

$$
\begin{aligned}
& \mathbf{E}+\frac{\mathbf{u}_{e}}{c} \times \mathbf{B}=\mathbf{R}_{e} \equiv+\left[\frac{1}{n e} \nabla \cdot \mathbf{P}_{e}\right]_{\text {pressure }}+\left[\frac{-1}{e} \frac{D}{D t}\left(m_{e} \mathbf{u}_{e}\right)\right]_{\text {inertial }} \\
& \mathbf{E}+\frac{\mathbf{u}_{i}}{c} \times \mathbf{B}=\mathbf{R}_{i}=\left[\frac{\mathbf{J}}{n e c} \times \mathbf{B}\right]_{\text {Hall }}+\mathbf{R}_{e}, \quad \mathbf{V}_{\mathrm{MHD}} \approx \mathbf{u}_{i}
\end{aligned}
$$

Equation (3a) is the exact electron momentum equation. By adding and subtracting the ion Lorentz force from Eq. (3a) and rearranging terms one obtains Eq. (3b), which is the generalized Ohm's law for the (Hall) ion fluid. The ion fluid is essentially the same as the MHD fluid since the MHD single-fluid flow velocity, $\mathbf{V}_{\mathrm{MHD}}=\mathbf{u}_{i}+\left(m_{e} / M_{i}\right) \mathbf{u}_{\mathbf{e}}$ is mainly carried by ions. The first term on the right in Eq. (3b) is the Hall term, proportional to the force density, $\mathbf{J} \times \mathbf{B}$. The Hall term and related physics are always automatically included in kinetic (particle-in-cell) simulations of reconnection (Drake et al. 2008).

In the Hall model the magnetic field, $\mathbf{B}$, is frozen-in to the electron fluid. This means $\mathbf{R}_{e}$ is negligible on the right side of Eq. (3a), so $\mathbf{E}_{\perp}=-\mathbf{u}_{e \perp} \times \mathbf{B}$. The Hall model assumes $\left|\mathbf{u}_{e \perp}\right| \gg\left|\mathbf{u}_{i \perp}\right|$, as PIC reconnection simulations show is often the case in certain (ion diffusion) regions. In these regions $\mathbf{J}_{\perp}$ is dominated by the electron current. Since $\left|\mathbf{E}_{\perp}\right|=\left|\mathbf{u}_{e} \times \mathbf{B}\right| \gg\left|\mathbf{u}_{i \perp} \times \mathbf{B}\right|$, the ions are non-ideal and the perpendicular electric field, $\mathbf{E}_{\perp}$ is effectively the Hall term on the right side of the generalized Ohm's law, which therefore cannot be neglected:

$$
\mathbf{E}_{\perp} \approx[\mathbf{J} \times \mathbf{B} /(n e c)], \quad \text { provided } \mathbf{E}+\mathbf{u}_{e} \times \mathbf{B}=0 \quad \text { and } \quad\left|\mathbf{u}_{e \perp}\right| \gg\left|\mathbf{u}_{i \perp}\right|
$$

Regions where measurements of the field $\mathbf{E}_{\perp}$ reveal that Eq. (4a) is not satisfied must effectively be electron diffusion regions, where electrons are not frozen-in (see, for example, Angelopoulos et al. 2013).

In regions where Eq. (4a) is satisfied, the field $\mathbf{E}_{\perp}$ is usually called the Hall electric field, $\mathbf{E}_{H}$. The quadrupole Hall magnetic field, $\mathbf{B}_{H}$ is in the $\pm z$-direction ( \pm GSM- $y$ ). In one explanation of the origin of $\mathbf{B}_{H}$, anti-parallel electron currents, $\mathbf{J}_{\mathbf{e}=}$, form on either side of the separatrices due to flux-tube-widening (Uzdensky and Kulsrud 2006) and anti-mirroring of electrons. As a result of Faraday's Law, these in-plane currents (sometimes called Hall currents) produce a quadrupolar Hall magnetic field, $\mathbf{B}_{H}=\mathbf{B}_{z}$ in the $\pm z$ directions for $\mathbf{B}_{g}=0$ and a distorted quadrupolar magnetic field for non-zero guide field, $0<B_{g} \ll B_{0}$. Hall fields in the presence of a guide field have been found in simulations (Pritchett and Coroniti 2004) and measured in the magnetotail (Eastwood et al. 2010a, 2010b) As mentioned earlier a guide field of $B_{g}=1 \mathrm{nT}$ is not uncommon in the magnetotail and simulations which include this small guide field or an equivalent lead to effects which are not captured in antiparallel PIC simulations. The guide and Hall magnetic fields add together to give $\mathbf{B}_{z}=\mathbf{B}_{g}+\mathbf{B}_{H}$; the complete local magnetic field is $\mathbf{B}=\mathbf{B}_{=}+\mathbf{B}_{z}$. The Hall magnetic field is contained in $\mathbf{B}_{z}$, although observationally the guide-field, $\mathbf{B}_{g}$, cannot always be identified separately from $\mathbf{B}_{H}$ in $\mathbf{B}_{y \text {-GSM }}$. 

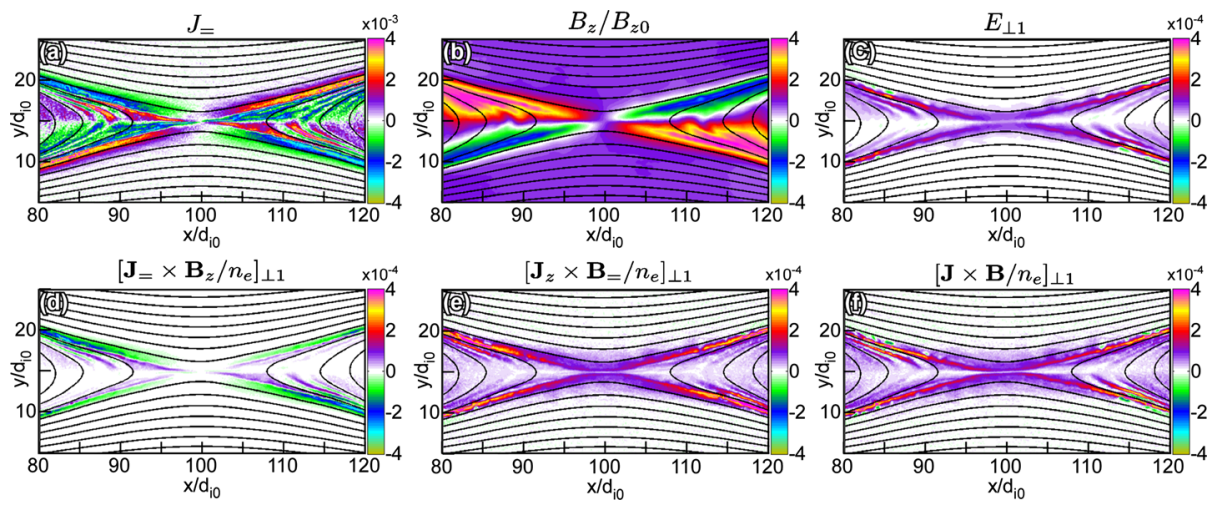

Fig. 8 Currents, fields and forces relevant to Hall physics from Simulation A. Coordinate systems are as defined in Fig. 6. (a) In-plane current, $J_{=}$, (b) $B_{z}=B_{H}+B_{g}$, (c) In-plane, $E_{\perp 1}$ defined as positive when vector $\mathbf{E}_{\perp 1}$ is rotated clockwise $90^{\circ}$ from direction of local magnetic field line. (c), (d) Two parts of $(\mathbf{J} \times \mathbf{B})_{\perp 1} /($ nec $)$ : from $\mathbf{J}_{=}$and from $\mathbf{J}_{z}$, (e) Sum of those two parts

Figure $8 \mathrm{a}$, shows the parallel in-plane electron (Hall) current, $J_{=}$, and Fig. $8 \mathrm{~b}$ shows the weak guide-field-distorted quadrupolar geometry of $\mathbf{B}_{z}$ in Simulation A (with $B_{g}=0.1 B_{0}$ ). Near the separatrices, where the ions are demagnetized, the ions don't significantly contribute to $\mathbf{J}_{=}$. The parallel in-plane Hall electron current, $J_{=}$is positive or negative (parallel or antiparallel to $\mathbf{B}_{=}$), depending on its location above or below the separatrix. This is especially pronounced in the upper right quadrant $(x, y>0)$ in Fig. 8a where the red, yellow and violet regions outside the separatrix indicate current directed away from the $x$-pt. while the green and blue regions inside indicate current directed towards the $x$-point.

In order to better understand the Hall electric field, $\mathbf{E}_{H}$, we expand the Hall term into three parts: $t$ wo from the forces associated with the out-of-plane magnetic field $\mathbf{B}_{z}: \mathbf{J}_{=} \times \mathbf{B}_{z}$ and $\mathbf{J}_{\perp 1} \times \mathbf{B}_{z}$, and one from the force associated with the in-plane magnetic field, $\mathbf{B}_{=}$: $\mathbf{J}_{z} \times \mathbf{B}_{=}$.

$$
\mathbf{J} \times \mathbf{B}=\left(\left[\mathbf{J}_{=} \times \mathbf{B}_{z}\right]+\left[\mathbf{J}_{z} \times \mathbf{B}_{=}\right]\right)_{\perp 1}+\left(\mathbf{J}_{\perp 1} \times \mathbf{B}_{z}\right)_{\perp 2}
$$

The last term on the right is small because $\mathbf{J}_{\perp 1}$, is small over regions of interest for Hall fields. Hence, $\mathbf{J} \times \mathbf{B}$ points mainly in the $\perp 1$ direction in such regions. Plotted in Fig. 8d, 8e are the values of $\left[\mathbf{J}_{=} \times \mathbf{B}_{z}\right]_{\perp 1} /$ (nec) and $\left[\mathbf{J}_{z} \times \mathbf{B}_{=}\right]_{\perp 1} /$ (nec) from Simulation A. The sum is plotted in Fig. 8f. Comparing Fig. 8f with Fig. 8c, it is evident that $(\mathbf{J} \times \mathbf{B})_{\perp 1} /($ nec $)$ is a good approximation to the independently-calculated simulation value for the component of $\mathbf{E}$ in the $\perp$ 1-direction shown in Fig. 8c:

$$
E_{\perp 1} \approx\left[\mathbf{J}_{=} \times \mathbf{B}_{z}\right]_{\perp 1} / \text { nec }
$$

This is consistent with frozen-in electrons. The vector field $\mathbf{E}_{\perp 1}$ points from each separatrix towards the center of the exhaust. In Hall MHD, in which lines of $\mathbf{B}$ are frozen-in to the electrons (Huba and Rudakov 2004; Huba 2005) the Hall electric field, $\mathbf{E}_{H}$ is defined as $[\mathbf{J} \times \mathbf{B} /($ nec $)]$ in Eq. (4a). However, in more general Hall-effect applications $\mathbf{E}_{H}$ is interpreted as only the (smaller-magnitude) $\left[\mathbf{J}_{=} \times \mathbf{B}_{z} /(\right.$ nec $\left.)\right]$ part of $[\mathbf{J} \times \mathbf{B} /($ nec $)]$. This is the part that is the cross-product of the Hall current and the Hall magnetic field, as in the standard Hall effect in physics. Even for reconnection with a small guide field, the contribution, 
$\left[\mathbf{J}_{=} \times \mathbf{B}_{z} /(n e c)\right]$ exhibits striations of different sign, as seen in Fig. 8d. The associated striations may be related to kinetic Alfven waves discussed in anti-parallel reconnection (Shay et al. 2011).

Both $[\mathbf{J} \times \mathbf{B} /($ nec $)]$ and the dominant contribution to it, $\mathbf{J}_{z} \times \mathbf{B}_{=} /($nec $)$, are mainly electrostatic fields (Wygant et al. 2005; Zhi-Wei and Shu-Ling 2008) associated with charge separation near the separatrix. In traditional physics Hall effects the Hall electric field is also electrostatic and due to charge-separation. One argument for why the Hall electric field defined as $\mathbf{J} \times \mathbf{B} /($ nec $)$ is electrostatic in the ion diffusion region relies on Ampere's law (without displacement current) to express $\mathbf{J} \times \mathbf{B} /($ nec $)$ as

$$
\mathbf{E}_{H}=\mathbf{J} \times \mathbf{B} /(n e c)=\left\{-\nabla\left(B^{2} / 2\right)+\mathbf{B} \cdot \nabla \mathbf{B}\right\} /(4 \pi n e) .
$$

The first term on the right is proportional to the gradient of the magnetic energy density and the second to the magnetic stress of curved field lines. In a region where $\mathbf{B}$-lines are fairly straight and the density is fairly uniform, the gradient term dominates and $\mathbf{E}_{H}$ is electrostatic, with potential, $B^{2} / 8 \pi n e$ (Wygant et al. 2005). The Hall electric field, $\mathbf{E}_{H}$ in Eq. (4d) is often the strongest electric field measured during tail reconnection. In physical units, using $B_{0}=10 \mathrm{nT}$ (twice $B_{x}$ on the low density side of the Themis event in Fig. 3), the simulation Hall electric field has magnitude $E_{\perp 1}=10^{-4}$, which translates into a physical Hall electric field of $E_{\mathrm{GSM}-z}=10^{-4} \cdot 100 \cdot\left[\left(3 \times 10^{8}\right) \cdot\left(10 \times 10^{-9}\right)\right]=30 \mathrm{mV} / \mathrm{m}$. Hall electric fields of this magnitude and larger have been measured (Wygant et al. 2005).

\subsection{Measures of Diffusion Regions}

PIC simulations reveal that topological reconnection occurs in electron diffusion regions and that the rate of reconnection can be affected by turbulence or other physical conditions near this region. This is why a major goal of MMS is to probe electron diffusion regions. However, the electron diffusion region around the $x$-point is relatively small and it is not the region where significant transfer of magnetic energy to particle energy occurs. Both Poynting flux and electron and ion energy fluxes carry energy out of this region. It has been judged unlikely that MMS will cross into the electron diffusion region in the magnetotail more than once or twice during the entire mission lifetime (S. Fuselier, private communication). However, estimates of the number of MMS crossings of the ion diffusion region in the magnetotail are an order of magnitude higher. Diffusion regions in two-dimensional reconnection simulations are easier to interpret than in 3D simulations because a boundary surface between unreconnected and reconnected field lines, the magnetic separatrix, exists in 2D reconnection. This paper is limited to interpretations of magnetic reconnection found in 2D PIC simulations although there have been recent simulations of 3D reconnectionboth symmetric (i.e., magnetotail) and asymmetric (i.e., dayside) (Daughton et al. 2014). Common theoretical measures of diffusion regions include the following:

(i) Slippage. From the discussion of Eqs. (2a)-(2i) if all components of $\mathbf{R}=\mathbf{E}+\mathbf{u} \times \mathbf{B}$ vanish for electrons or ions in a spatial region then the corresponding magneto-fluid is ideal in that region and the magnetic field is frozen-in. But is the converse true? Is a region where $\mathbf{R}=\mathbf{E}+\mathbf{u} \times \mathbf{B} \neq 0$ a diffusion region, and what is the correct measure of "not equal to zero?" As in the discussion below Eqs. (2g) and (2f), if $\mathbf{E}+\mathbf{u} \times \mathbf{B}=$ $\nabla \psi$, then magnetic flux and magnetic field lines are both conserved so topological reconnection cannot occur and such a region technically should not be called a diffusion region! First we assume that the deviation of $\mathbf{R}=\mathbf{E}+\mathbf{u} \times \mathbf{B}$ from zero is not a gradient and that magnetic flux is not conserved (i.e., $\nabla \times(\mathbf{E}+\mathbf{u} \times \mathbf{B}) \neq 0)$. Only one of the 
components of $\mathbf{R}$ need deviate significantly from zero. Consider one commonly used measure of a diffusion region for species $\mathrm{s}$ in which one of the two components of $\mathbf{R}_{\perp s}$ deviates significantly from zero. One can express this condition in terms of the difference of at least one of the components of the perpendicular velocity, $\mathbf{u}_{\perp s}$, from the $\mathbf{E} \times \mathbf{B} / B^{2}$ drift velocity:

$$
\begin{aligned}
& \delta \mathbf{u}_{\perp s}=\left[\mathbf{u}_{\perp s}-\mathbf{E} \times \mathbf{B} / B^{2}\right]=\mathbf{R}_{s} \times \mathbf{B} / B^{2} \neq 0 \\
& \Rightarrow \quad \text { at least one component } \neq 0, \\
& \delta u_{\perp 1 s}= {\left[u_{\perp 1 s}-E_{\perp 2} / B\right]=R_{2 s} / B \neq 0, \quad \text { or } } \\
& \delta u_{\perp 2 s}=\left[u_{\perp 2 s}+E_{\perp 1} / B\right]=-R_{1 s} / B \neq 0
\end{aligned}
$$

These conditions say that at least one component of $\mathbf{u}_{\perp s}$ is (significantly) slower or faster than the $\mathbf{E} \times \mathbf{B}$ drift velocity. Equation (5a) has been called (Schindler et al. 1991) the slippage condition for species, $s$. As defined here, the slippage is relative to the drift velocity, which is not the velocity of magnetic field lines because neither flux nor field lines are conserved. A significant departure of $\left|\delta \mathbf{u}_{\perp s}\right|$ from zero in Eq. (5a), (5b) and (5c) might be a difference on the order of the local Alfven speed of species $s, v_{s A}$. An alternative "minimum measure" of $\left|\delta \mathbf{u}_{\perp s}\right|$ which satisfies Eq. (5a), (5b) and (5c) is the magnitude of $\left|\mathbf{E} \times \mathbf{B} / B^{2}\right|$.

In regions where Hall physics dominates, the electrons are frozen-in while the slower ions slip. In Hall regions $\mathbf{R}_{i}=\mathbf{E}+\mathbf{u}_{i} \times \mathbf{B}=\mathbf{J} \times \mathbf{B} /($ nec $)$ is essentially a gradient if the magnetic field lines are straight and the density is uniform (see Eq. (4d)). In this limit the curl of $\mathbf{R}_{i}$ vanishes so one must be careful about equating ion "slippage" with flux conservation!

For the same reason, slippage due to a term in $\mathbf{R}_{s}$ which is a scalar pressure gradient, does not break conservation of both flux and lines. In this case the right side of (5a) can be interpreted as a diamagnetic drift. In other words, a generalized slippage condition can still be satisfied in which fluid elements are moving at a "drift" velocity, but that "drift" is not the $\mathbf{E} \times \mathbf{B}$ drift.

(ii) Diffusion region defined by explicit violation of magnetic flux conservation. A reconnection rate (time rate of change of flux) can exist only when magnetic flux is not conserved. A fundamental measure of a diffusion region is the violation of the magnetic flux conservation expressed in Eqs. (2h), (2i) (Newcomb 1958; Vasyliunas 1972, 1975):

$$
\nabla \times\left[\mathbf{E}+\mathbf{u}_{s} \times \mathbf{B}\right]=\nabla \times \mathbf{R}_{s} \neq 0
$$

In terms of parallel and perpendicular components, Eq. (6a) may be written as

$$
\begin{aligned}
& \left(\nabla \times\left[\mathbf{E}+\mathbf{u}_{s} \times \mathbf{B}\right]\right)_{\|} \neq 0 \text { or } \\
& \left(\nabla \times\left[\mathbf{E}+\mathbf{u}_{s} \times \mathbf{B}\right]\right)_{\perp} \neq 0
\end{aligned}
$$

Even when there is no slippage, i.e., when $\mathbf{E}_{\perp}+\mathbf{u}_{s} \times \mathbf{B}=0$ (so that $\left|\delta \mathbf{u}_{\perp}\right|=0$ ), Eq. (6a) implies that a parallel electric field, $\mathbf{E}_{\|}$alone can cause violation of flux conservation:

$$
\nabla \times\left[\mathbf{E}+\mathbf{u}_{s} \times \mathbf{B}\right]=\nabla \times \mathbf{E}_{\|} \neq 0
$$


Even in regions where there is no slippage (i.e., where (Eq. (6c)) is satisfied) line conservation (Eq. (2g)) can be violated as well as magnetic flux conservation. Equation (6c) is often written as,

$$
\mathbf{B} \times\left(\nabla \times\left[\mathbf{E}+\mathbf{u}_{s} \times \mathbf{B}\right]\right) \neq 0
$$

Once again, if $\mathbf{E}_{\perp}+\mathbf{u}_{s} \times \mathbf{B}=0$ (i.e., there is no slippage), this can be expressed as,

$$
\mathbf{B} \times\left(\nabla \times \mathbf{E}_{\|}\right) \neq 0
$$

An alternative way of writing Eq. $\left(6 \mathrm{~d}^{\prime}\right)$ (derived by the authors) is:

$$
B\left[\nabla_{\perp} E_{\|}-E_{\|} \hat{\boldsymbol{b}} \cdot \nabla \hat{\boldsymbol{b}}\right] \neq 0
$$

The second term vanishes for straight magnetic field lines. It is important to note, however that even when magnetic field lines are preserved in a region (i.e., $\mathbf{B} \times(\nabla \times$ $\left.\left[\mathbf{E}+\mathbf{u}_{s} \times \mathbf{B}\right]\right)=0$, it is possible that Eq. (6b) is satisfied, so that flux conservation is still violated. It appears, therefore, that violation of flux conservation (Eq. (6a)) is the more fundamental criterion for a diffusion region.

(iii) Work and transport equations. Still another signature of a non-frozen-in region for electrons or ions is a region where significant \pm work is performed by the local electric field $\mathbf{E}$ on the electron or ion current densities, $\mathbf{J}_{e}$ and $\mathbf{J}_{i}$. Transport equations for the electron or ion fluids are associated with work done on the electron or ion currents. Work done by the total current density, J, determines transport of field energy density. The (collisionless) energy transport equations are most easily derived from the Maxwell and Vlasov equations for distribution functions $f_{s}(\mathbf{v})$, where $s=e$ for electrons or $i$ for ions:

$$
\begin{aligned}
& -\mathbf{E} \cdot \mathbf{J}=\nabla \cdot \mathbf{S}+\frac{\partial U_{\text {field }}}{\partial t}, \quad \mathbf{S}=\frac{\mathbf{E} \times \mathbf{B}}{4 \pi}, \quad U_{\text {field }}=\frac{B^{2}+E^{2}}{8 \pi} \\
& \mathbf{E} \cdot \mathbf{J}_{s}=\nabla \cdot \mathbf{Q}_{s}+\frac{\partial U_{s}}{\partial t}, \quad \mathbf{Q}_{s}=n_{s}\left\langle\mathbf{v} \frac{m_{s} v^{2}}{2}\right\rangle, \\
& \langle\mathbf{G}(\mathbf{v})\rangle \equiv \frac{1}{n_{s}} \int d^{3} \mathbf{v} f_{s}(\mathbf{v}) \mathbf{G}(\mathbf{v}), \quad s=e, i
\end{aligned}
$$

Equation (7a) says that the work, $-\mathbf{E} \cdot \mathbf{J}$ performed by the total current $\mathbf{J}$ on $\mathbf{E}$ results in time variation of field energy density, $U_{\text {field }}$, and/or transport of field energy density by Poynting flux, $\mathbf{S}$. Another common measure of a diffusion region is $\mathbf{E} \cdot \mathbf{J} \neq 0$. When $\mathbf{E}$. $\mathbf{J} \neq 0$, magnetic field transport can take magnetic energy out of flux tubes, potentially violating flux-conservation.

Measures of the separate electron and ion diffusion regions are given by $\mathbf{J}_{s} \cdot \mathbf{E} \neq 0$ for $s=e$ or $i$. Equation (4b) says that the work, $\mathbf{E} \cdot \mathbf{J}_{s}$ done by $\mathbf{E}$ on the current $\mathbf{J}_{s}$ carried by species $s$ results in a time variation of the energy density, $U_{s}$ of species $s$ and/or transport of particle energy density by a total energy flux, $\mathbf{Q}_{s}$, for each species. Both of these quantities are simple velocity moments of the distribution function, $f_{s}$, of species $s$ which can be computed from MMS particle data.

The energy density $U_{s}$ is a sum of bulk flow energy and thermal energy for species, $s$.

The total energy flux for electrons or ion, $\mathbf{Q}_{s}$, is a direct particle analogue of the Poynting flux in the electromagnetic energy transport equations. It is the sum of the flux 
of bulk flow energy, the enthalpy flux and the heat flux of species $s$. In Eq. (8a)-(8e) the terms are broken out explicitly. $\mathbf{Q}_{s 1}$ is the energy flux of the bulk flow, $\mathbf{Q}_{s 2}+\mathbf{Q}_{s 3}$ gives the two terms constituting the enthalpy flux expressed in terms of the pressure tensor, $\mathbf{P}$, and $\mathbf{q}_{s}$ is the heat flux:

$$
\begin{aligned}
& \mathbf{Q}_{s}=\mathbf{Q}_{s 1}+\mathbf{Q}_{s 2}+\mathbf{Q}_{s 3}+\mathbf{q}_{s} \\
& \mathbf{Q}_{s 1}=\frac{n_{s} m_{s} u_{s}^{2}}{2} \mathbf{u}_{s} \\
& \mathbf{Q}_{s 2}=\frac{\operatorname{Tr} \overrightarrow{\vec{P}}_{s}}{2} \mathbf{u}_{s}, \quad \mathbf{Q}_{s 3}=\mathbf{u}_{s} \cdot \overrightarrow{\overrightarrow{\mathbf{P}}} \\
& \overrightarrow{\overrightarrow{\mathbf{P}}}_{s} \equiv n_{s} m_{s}\left\langle\delta \mathbf{v}_{s} \delta \mathbf{v}_{s}\right\rangle \\
& \mathbf{q}_{s} \equiv \frac{n_{s} m_{s}}{2}\left\langle\delta \mathbf{v}_{s}\left|\delta v_{s}\right|^{2}\right\rangle
\end{aligned}
$$

Note that if the velocity of species- $s$ is frozen-in according to the criterion $[\mathbf{E}+$ $\left.\mathbf{u}_{s} \times \mathbf{B}\right]=0$, then it follows mathematically from $\mathbf{J}_{s}=q_{s} n_{s} \mathbf{u}_{s} \propto(\mathbf{E} \times \mathbf{B})$ that $\mathbf{J}_{s} \cdot \mathbf{E}=0$.

In general, by adding Eq. (7b) for each species to Eq. (7a) all $\mathbf{J} \cdot \mathbf{E}$ work terms are eliminated and one arrives at a continuity equation for field plus particle energies and fluxes:

$$
\partial_{t}\left(U_{\text {field }}+U_{i}+U_{e}\right)+\nabla \cdot\left(\mathbf{S}+\mathbf{Q}_{i}+\mathbf{Q}_{e}\right)=0
$$

The criterion $\mathbf{J}_{s} \cdot \mathbf{E} \neq 0$ for electrons and for ions will be explored and evaluated as signatures of the (separate) electron and ion diffusion regions and compared with other measures found in our 2D PIC simulations. The work expressions in Eqs. (7a) and (7b) will all be evaluated in the frame of the simulation. They are not frame-invariant, so MMS data will have to be transposed to this frame for comparison. Since the effective frame of spacecraft measurements is often determined by motions of the magnetotail such as flapping, it would be useful to have a work criterion that is frame independent. Such a "work" criterion has been suggested (Zenitani et al. 2011):

$$
D_{e}=\mathbf{J} \cdot\left[\mathbf{E}+\mathbf{u}_{e} \times \mathbf{B}\right]-\rho \mathbf{u}_{e} \cdot \mathbf{E} \neq 0
$$

Here, $\mathbf{u}_{e}$ is the electron flow velocity, which varies with position. We shall see from our 2D simulations that condition (7d) can signal the presence of an electron diffusion region. The last term on the right side of (7d) is present because a frame transformation can cause a stationary net charge distribution, $\rho$, to become a current. That term is usually small compared to the others. When that term is negligible we have a new interpretation of $D_{e}$ which will be described later (Sect. 3.3)

(iv) Kinetic particle distribution signatures of diffusion regions. MMS will be able to provide highly resolved (i.e., time-averaged over a time interval of $30 \mathrm{~ms}$ instead of $3 \mathrm{sec}$ ) electron and ion distributions which can provide additional ways to identify diffusion regions using kinetic (PIC) simulations which also provide particle distributions. Agyrotropy of a particle distribution (in the plane whose normal is parallel to the local magnetic field, B) is a kinetic indication that a species is not frozen-in. A gyrotropic distribution has azimuthal symmetry about $\mathbf{B}$ (in a frame in which the velocity moment vanishes). An agyrotropic (or nongyrotropic) velocity distribution has no azimuthal symmetry about $\mathbf{B}$ in any frame. Agyrotropy in a species can be the source of offdiagonal terms in the local pressure tensor moment for that species. Studies of electron 
agyrotropy based on analysis of the electron pressure tensor moments have been carried out in 2-D Harris sheet simulations of reconnection (Aunai et al. 2013, and references therein). Contributions to the generalized Ohm's law from such off-diagonal terms to the $\nabla \cdot \mathbf{P}$ pressure force term in $\mathbf{R}_{s}$ can cause slippage $\left[\mathbf{E}+\mathbf{u}_{s} \times \mathbf{B}\right]_{\perp} \neq 0$, as well as violating flux conservation. However, MMS (by means of the Fast Plasma Instrument) should be able to construct full electron velocity space distributions in $15 \mathrm{~ms}$, allowing direct measurement of agyrotropy which should facilitate kinetic theory explanations for agyrotropy.

(v) Influence of wave turbulence on diffusion regions. Waves and turbulence can affect the reconnection rate by exerting effective forces capable of changing electron momentum. Among the various waves and instabilities found in simulations of magnetic reconnection are:

(a) Electron-ion (Buneman) instabilities (Drake et al. 2003) and electron-electron two-stream instabilities which saturate by trapping electrons and forming electron phase-space holes (parallel bipolar electrostatic fields) near magnetic separatrices (Goldman et al. 2014; Lapenta et al. 2011). Holes can create an anomalous drag term in the electron momentum equation but do not appear to significantly speed up the reconnection rate. Holes have been measured during magnetotail reconnection (Cattell et al. 2005; Deng et al. 2010) and the magnitude of the associated bipolar parallel electric fields agrees well with $2 \mathrm{D}$ simulations.

(b) Electromagnetic whistler waves near the $x$-line which can be Čerenkov emitted by fast electron phase-space holes and modulate the reconnection rate (Goldman et al. 2014).

(c) A possible whistler-like shear-instability of thin current sheets in a magnetic guide field, $\mathbf{B}_{g}$, (Drake et al. 1994; Che et al. 2011) which causes the current sheet to broaden and leads to anomalous diffusivity in the generalized Ohm's law. This diffusivity has been linked to faster reconnection rates although the existence of the instability is still controversial (Liu et al. 2013).

(d) Kelvin-Helmholtz instabilities in thin sheared current sheets may also lead to reconnection (Faganello et al. 2010; Lapenta et al. 2014).

(e) An ion firehose instability driven in the ion-diffusion region by counterstreamingion-beams in the ion-diffusion region during anti-parallel reconnection (Liu et al. 2013). The ion-beams effectively increase the ion pressure parallel to the local $\mathbf{B}$ in comparison to the perpenciular pressure, thus moving the firehose parameter into the unstable range.

(vi) Spacecraft measures of diffusion regions. It is useful to understand observational measures of diffusion regions although our focus in this paper is largely on theory. For example, a method commonly used onboard spacecraft to help identify ion diffusion regions is to look for reversals in both the outflow, $u_{i x}$, and $B_{\mathrm{GSM}-z}$ when traversing the exhausts on both sides of the $x$-point (Eastwood et al. 2010a, 2010b; Eastwood, private communication).

In addition measurements showing deviations of $\mathbf{E}_{\perp}+\mathbf{u}_{i} \times \mathbf{B}$ from zero have been used to identify ion diffusion regions and deviations of $\mathbf{E}_{\perp}+\mathbf{u}_{i} \times \mathbf{B}$ from the Hall term $\mathbf{J} \times \mathbf{B} /$ ne have been used to find electron diffusion regions (see Eq. (4a) and Angelopoulos et al. 2013). It is often assumed that $E_{\|}$is negligible so that curl $\mathbf{E}_{\|}$ would also be negligible. (See above section on slippage for associated subtletiesparticularly in interpretations of ion slippage in regions of Hall physics.) 

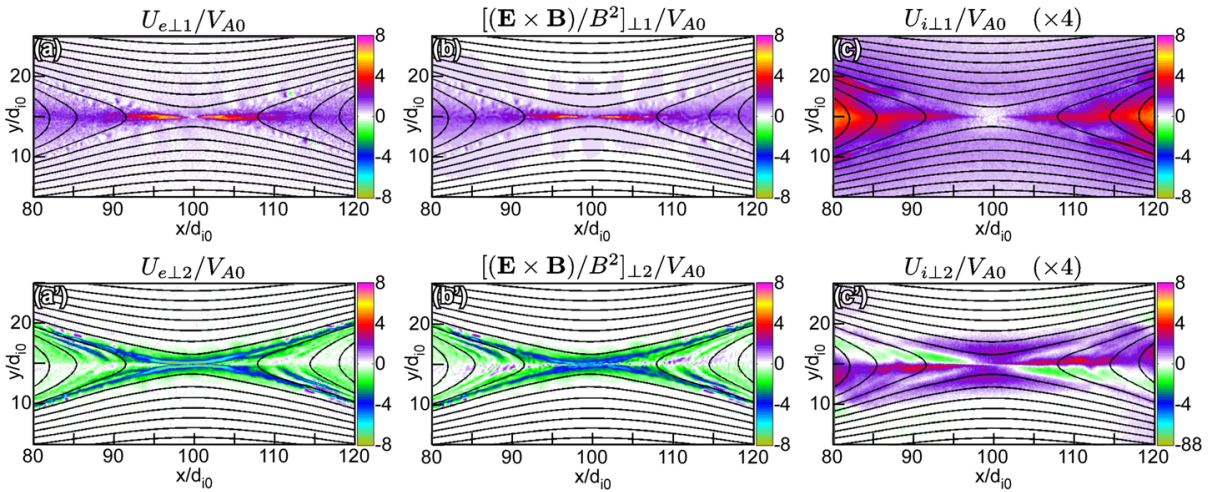

Fig. 9 Flow velocities compared to drift velocity from Simulation A, normalized to the Harris Alfven velocity, $v_{A 0}$ : 1st column, top to bottom: (a) electron flow velocity, $u_{e \perp 1},\left(\mathbf{a}^{\prime}\right) u_{e \perp 2} ;$ (b) drift velocity component, $\left[\mathbf{E} \times \mathbf{B} / B^{2}\right]_{\perp 1},\left(\mathbf{b}^{\prime}\right)\left[\mathbf{E} \times \mathbf{B} / B^{2}\right]_{\perp 2} ;(\mathbf{c})$ four times ion flow velocity, $u_{i \perp 1},\left(\mathbf{c}^{\prime}\right)$ four times $u_{i \perp 2}$

\section{Identification of Diffusion Regions in Reconnection Simulations}

In this section various measures for identifying diffusion regions are applied to simulations of tail reconnection. The main simulation studied is Simulation A, at time $t=30 \Omega_{i}^{-1}$.

\subsection{Simulation Results for Diffusion Regions in Terms of Slippage}

In Fig. 9 electron and ion perpendicular flow velocities, $u_{s \perp 1}$ and $u_{s \perp 2}$ are compared with the drift velocities, $(\mathbf{E} \times \mathbf{B})_{\perp 1,2} / B^{2}$, with all velocities in units of the Harris Alfven velocity $v_{A 0}$. Figures $9 \mathrm{a}$ and $9 \mathrm{a}^{\prime}$ show the components of $\mathbf{u}_{e \perp}$, the electron perpendicular flow velocity, which should be compared with the components of $(\mathbf{E} \times \mathbf{B}) / B^{2}$ in Figs. $9 \mathrm{~b}$ and $9 \mathrm{~b}^{\prime}$. The agreement is good in most regions, indicating that the electron fluid is moving at the drift velocity so there is no slippage or overtaking of the drift velocity.

The exception is a region in $u_{e \perp 2}(y)$ near the $x$-point $(y=15, x=100)$, where a more detailed examination reveals that $\left|u_{e \perp 2}(y)\right|$ exceeds $\left|\mathbf{E} \times \mathbf{B} / B^{2}\right|$ by around fifty percent. The extent in $\pm \delta x$ about the $x$-pt. of this region is $\delta x \approx 5 d_{i 0}$, where $d_{i 0}$ is the usual unit of distance in simulations (the ion inertial length based on the Harris density, $n_{0}$ ). The halfwidth $\delta x$ can also be expressed in terms of $d_{e 0}$, the Harris electron inertial length, as $\Delta x \approx$ $80 d_{e 0}$. However the local density, near the $x$-line (see Fig. 1) is much lower than the Harris density, and given approximately by, $n_{\mathrm{loc}}=n_{0} / 20$, yielding a local electron inertial length, $d_{e \text {-loc }}=4.5 d_{e 0}$, which is well-resolved in the simulation. Hence, $\delta x=18 d_{e-\text { loc }}$. This is much larger than the width $\delta x=d_{e-\text { loc }} / 2$ sometimes used in "cartoon" sketches of the electron diffusion region about the $x$-pt. It should also be noted that some electron slippage extends to portions of the separatrices, where the electrons are usually said to be frozen-in.

Next, we examine the slippage of ions. Figures $9 \mathrm{c}$ and $9 \mathrm{c}^{\prime}$, show the ion flow velocities, $u_{\perp 1 i}$ and $u_{\perp 2 i}$, on a scale four times weaker than for electrons and drift velocities. In most regions $\left|\mathbf{u}_{\perp i}\right|$ is significantly smaller than the drift velocity, $\mathbf{v}_{D}=\mathbf{E} \times \mathbf{B} / B^{2}$, and $\left|u_{\perp 1 i}\right|$ is larger than $\left|u_{\perp 2 i}\right|$ so flux conservation is likely to be violated. (The asymmetry of the ion flow component $u_{\perp 2}$ above and below the $y=15$ axis is due to the small guide field in Simulation A.) The ions are significantly slower than the drift throughout most of the exhaust from the $x$-line to the beginning of the pile-up front both off and on the symmetry axis. This suggests that the ion slippage is caused by an $\mathbf{R}_{i}$ not everywhere proportional to a 

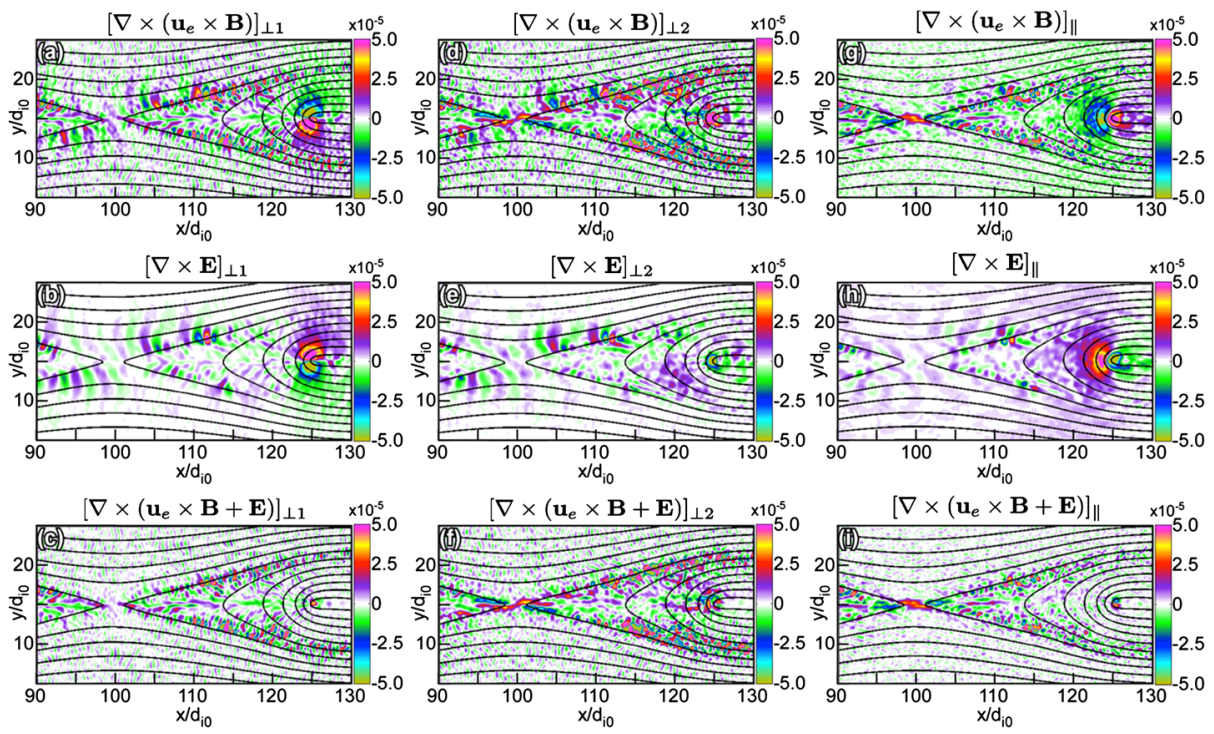

Fig. 10 Conservation of magnetic flux expression, $\nabla \times\left(\mathbf{E}+\mathbf{u}_{e} \times \mathbf{B}\right)$ decomposed into two pieces whose components are plotted together with the their sum: (a) $-(\mathbf{c}): \perp 1$ parts, $(\mathbf{d})-(\mathbf{f}): \perp 2$ parts, $(\mathbf{g})-(\mathbf{i}): \|$ parts

gradient, since magnetic curvature is pronounced on the symmetry axis (Eq. (4d)). Magnetic flux conservation is violated in these ion diffusion regions. It is not known whether this putative large ion diffusion region applies to physical magnetotail reconnection or is due to limitations in present-day simulation models.

\subsection{Regions of Magnetic Flux Nonconservation in Electron Fluid (from Simulations)}

In Fig. 10 details of the expression, $\nabla \times\left(\mathbf{E}+\mathbf{u}_{e} \times \mathbf{B}\right)$ used in the flux-non-conservation measure of electron diffusion regions, Eq. (6a), are analyzed by splitting the expression into two pieces and plotting the components of each piece, $[\nabla \times \mathbf{E}]_{j}$ and $\left[\nabla \times\left(\mathbf{u}_{e} \times \mathbf{B}\right)\right]_{j}$ together with $\left[\nabla \times\left(\mathbf{E}+\mathbf{u}_{e} \times \mathbf{B}\right)\right]_{j}$, for $\{j=\|, \perp 1$, or $\perp 2\}$. A sufficient condition for $\mid[\nabla \times(\mathbf{E}+$ $\left.\left.\mathbf{u}_{e} \times \mathbf{B}\right)\right]_{j} \mid$ to differ significantly from zero (i.e., for non-conservation of magnetic flux) is that $\left|\left[\nabla \times\left(\mathbf{E}+\mathbf{u}_{e} \times \mathbf{B}\right)\right]_{j}\right|>\left\{\right.$ either $\left|[\nabla \times \mathbf{E}]_{j}\right|$ or $\left.\left|\left[\nabla \times\left(\mathbf{u}_{e} \times \mathbf{B}\right)\right]_{j}\right|\right\}$. This is essentially the condition for a difference of two terms to have a larger magnitude than that of either term by itself.

Comparing Fig. 10i to Figs. 10g, 10h it is clear that there is an electron diffusion region around the $x$-point, where the contribution from $\left|[\nabla \times \mathbf{E}]_{\|}\right|$to $\left|\left[\nabla \times\left(\mathbf{E}+\mathbf{u}_{e} \times \mathbf{B}\right)\right]_{\|}\right|$is much smaller than the contribution from $\left|\left[\nabla \times\left(\mathbf{u}_{e} \times \mathbf{B}\right)\right]_{\|}\right|$. (The same appears to be true for the $\perp 2$ components in Figs. 10d, 10e, 10f.) There are indications in Fig. 10i of both a thin inner (warmer colors) electron diffusion region and a weak deflected thin outer (cooler colors) electron diffusion region. The inner region is a few $d_{i 0}$ from the $x$-line, while the outer (deflected) region extends out another few $d_{i 0}$, with very faint remnants (both positive and negative) out to $10 d_{i 0}$ from the $x$-line. The spatial extent of the electron diffusion region is much smaller than the extended electron diffusion regions found in antiparallel tail simulations and magnetosheath observations in the presence a guide field slightly larger than the guide field used in the present simulations (Phan et al. 2007). However, the smaller 

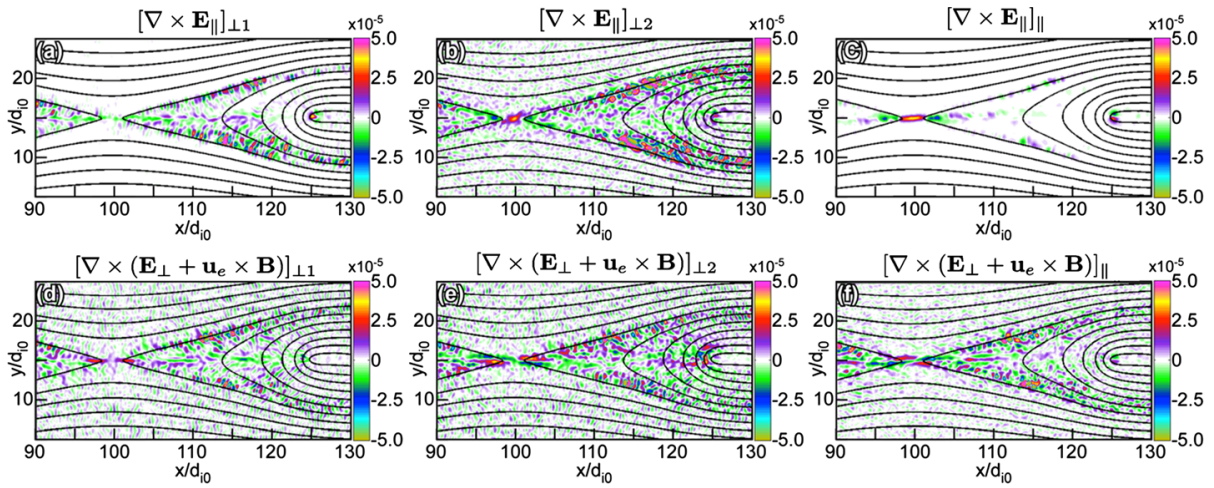

Fig. 11 Components of the two pieces $\nabla \times \mathbf{E}_{\|}$and $\nabla \times\left(\mathbf{E}_{\perp}+\mathbf{u}_{e} \times \mathbf{B}\right)$ whose sum is $\nabla \times\left(\mathbf{E}+\mathbf{u}_{e} \times \mathbf{B}\right)$. (a)-(c) $\perp 1, \perp 2$ and $\|$ components of $\nabla \times \mathbf{E}_{\|},(\mathbf{d})-(\mathbf{f}), \perp 1, \perp 2$ and $\|$ components of $\nabla \times\left(\mathbf{E}_{\perp}+\mathbf{u}_{e} \times \mathbf{B}\right)$

electron diffusion region found here is consistent with electron jets found in simulations using tail/magnetosheath parameters and a hydrogen mass ratio (Goldman et al. 2011). Recent kinetic simulations confirm this result (Le et al. 2013) but find more extended electron jets for a hydrogen mass ratio but with different simulation parameters from those of Goldman et al. (2011).

The pile-up region, by this measure, does not appear to be an electron diffusion region since there are large cancelling pieces in each component. However, the cancellation in the pile-up region is only marginal for the $\perp 2$ component, which should be studied in more detail. Interestingly, there are preliminary indications from 3D simulations (G. Lapenta, private communication) of an electron diffusion region in the pile-up region.

The separatrix region which contains electron phase space holes (Goldman et al. 2014) also satisfies the condition for instantaneous flux-non-conservation in the vicinity of the holes. However, the fine-spatial-scale alternations between positive and negative values needs to be averaged to interpret this properly, since the bipolar fields are moving (at speeds up to the electron Alfven speed). Any spacecraft measurement of $\mathbf{u}_{e}$ would be a timeaverage, which would greatly reduce the value of $\left\langle\left|\left[\nabla \times\left(\mathbf{E}+\mathbf{u}_{e} \times \mathbf{B}\right)\right]_{j}\right|\right\rangle_{t}$.

In Fig. 11 the same expression, $\nabla \times\left(\mathbf{E}+\mathbf{u}_{e} \times \mathbf{B}\right)$, used in the flux-non-conservation measure of electron diffusion regions, Eq. (6a), is decomposed in a different way by separately plotting the components of the two pieces $\nabla \times \mathbf{E}_{\|}+$and $\nabla \times\left(\mathbf{E}_{\perp}+\mathbf{u}_{e} \times \mathbf{B}\right)$ whose sum is $\nabla \times\left(\mathbf{E}+\mathbf{u}_{e} \times \mathbf{B}\right)$. As shown explicitly in Eq. (6d) and first derived by Newcomb (1958), the condition $\nabla \times \mathbf{E}_{\|} \neq 0$ indicates violation of conservation of flux and/or line-tying when perpendicular flow velocities are frozen-in. (The $\perp 1$ and $\perp 2$ components of $\nabla \times \mathbf{E}_{\|}$are the same as the $\perp 1$ and $\perp 2$ components of $\mathbf{B} \times\left(\nabla \times \mathbf{E}_{\|}\right) / B$.) By virtue of Eq. (2f) this requires that $\nabla \times\left(\mathbf{E}_{\perp}+\mathbf{u}_{e} \times \mathbf{B}\right)=0$.

\subsection{Simulation Results: Diffusion Regions in Terms of Work}

Another way to identify diffusion regions is to determine where work is done on particles by electric fields. In Fig. 12a and 12a' the $\mathbf{J}_{e} \cdot \mathbf{E}$ work done by electric fields on the electron current is plotted. An ("inner") electron diffusion region again seen in this diagnostic, with comparable half-width of the electron diffusion region, $\Delta x \cdot 4 d_{i 0} \cdot 14 d_{e \text {-loc }}$ as found from the slippage criterion (Fig. 9). Note random patchy regions outside the inner diffusion region, as in the flux-non-conservation diagnostic. These may be remanants of the exterior electron 

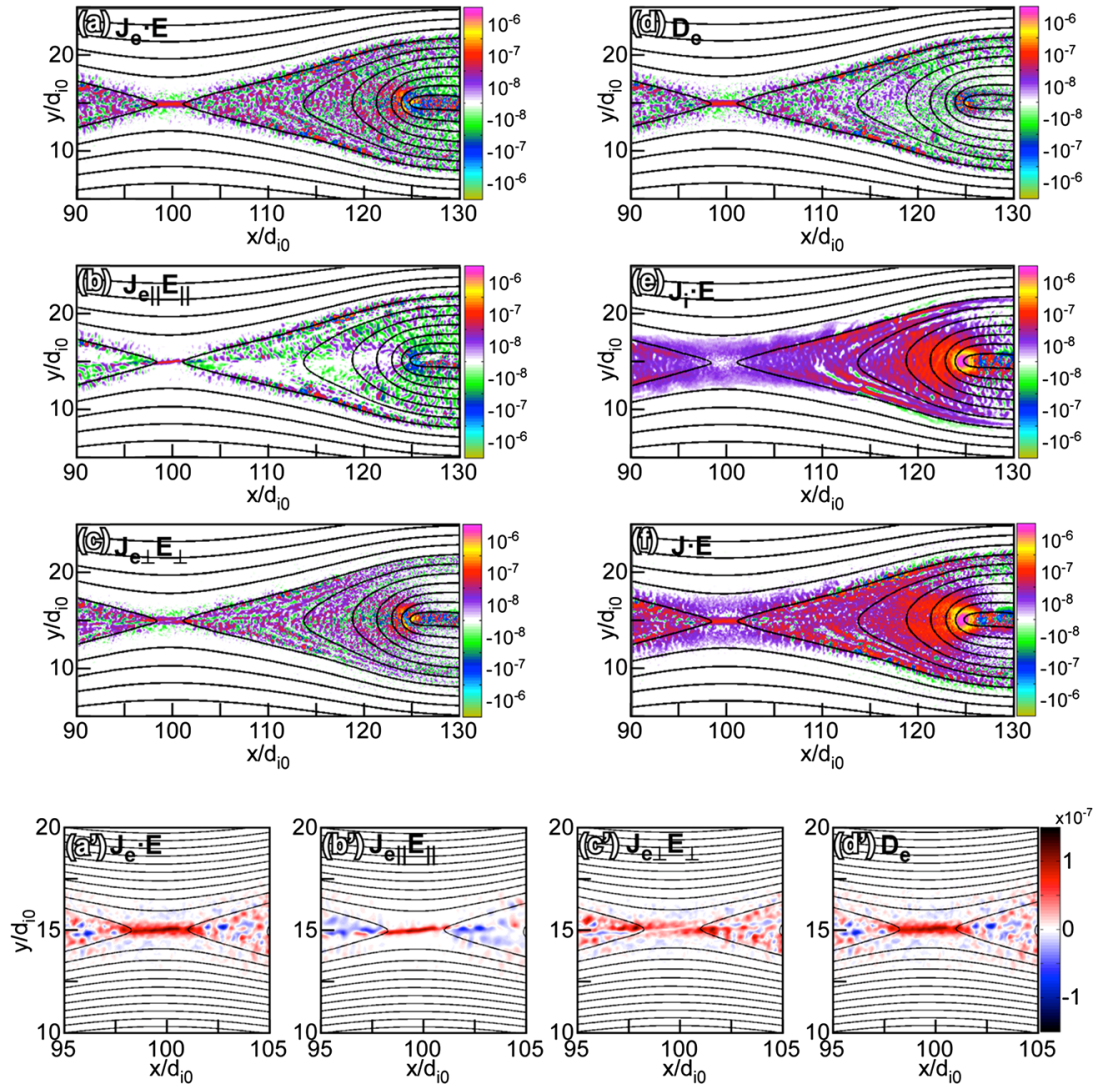

Fig. 12 Work performed on particles by electric fields (Simulation A). Signed-log color scale. (a) $\mathbf{J}_{e} \cdot \mathbf{E}$ work on electrons showing electron diffusion region, (b) Parallel part, $j_{e \|} E_{\|}$, showing parallel bipolar fields on sepa-ratrix, (c) Perpendicular part, $\mathbf{J}_{e \perp} \cdot \mathbf{E}_{\perp}$, (d) Diffusion measure, $D_{e}$, (Zenitani et al. 2011), (e) $\mathbf{J}_{i} \cdot \mathbf{E}$ work on ions showing ion diffusion region, (f) Total $\mathbf{J} \cdot \mathbf{E},\left(\mathbf{a}^{\prime}\right)-\left(\mathbf{d}^{\prime}\right)$ Blowups of (a)-(d) near $x$-point. There is no smoothing

jet diffusion region identified in antiparallel reconnection by Shay et al. (2007), but now disrupted because of the small guide field in the present reconnection simulations, as found by Goldman et al. (2011).

In Figs. 12b and 12c we have plotted separately the parallel and perpendicular pieces of the scalar product between $\mathbf{J}_{e}$ and $\mathbf{E}$ when it is decomposed into a sum of two scalar parts in $\mathbf{J}_{e} \cdot \mathbf{E}=J_{e \|} E_{\|}+\mathbf{J}_{\mathrm{e} \perp} \cdot \mathbf{E}_{\perp}$. There is a substantial contribution from $J_{e \|} E_{\|}$at the $x$-line and nearby because the small guide field allows an $E_{\|}$at the $x$-line, where the magnetic field is not frozen-into the electron fluid and we know that topological reconnection occurs. The plot of $J_{e \|} E_{\|}$in Figs. 12b, 12b' also shows that parallel electron phase space holes are manifested as (turbulent) bipolar parallel fields near the separatrices. Once again, even though the holes break the electron-fluid frozen-in condition instantaneously, the time-average, $\left\langle J_{e \|} E_{\|}\right\rangle_{t}$ will be greatly reduced in the vicinity of the holes because of the alternation of sign on a fine spatial scale.

The plot of $\mathbf{J}_{e \perp} \cdot \mathbf{E}_{\perp}$ also shows considerable turbulent behaviour in the upstream outflow region which is the major source of spottiness in the same region in the plot of $\mathbf{J}_{e} \cdot \mathbf{E}$ 
(Figs. 12a, 12a'). This may be associated with the breakup of the outer diffusion region due to the guide field or with other kinds of turbulence, such as kinetic Alfven waves (Shay et al. 2011). In addition, the plots of $\mathbf{J}_{e \perp} \cdot \mathbf{E}_{\perp}$ and of $\mathbf{J}_{e} \cdot \mathbf{E}$ show strong narrow peaks at the pile-up front at $x=123 d_{i 0}$ which are just as large as in the electron diffusion region. This is consistent with measurements by Angelopolous et al. (2013) who also find significant electron scale power conversion at the front and with recent 3D simulations by Vapirev et al. (2013).

In Fig. 12d the diffusion measure $D_{e}$ in Eq. (7d) (Zenitani et al. 2011) is plotted and seen to be similar to $\mathbf{J}_{e} \cdot \mathbf{E}$ out to five or ten $d_{i 0}$ from the $x$-point but different from $\mathbf{J}_{e} \cdot \mathbf{E}$ between $110 d_{i 0}$ and the pile-up front at $123 d_{i 0}$.

The work done on ions is displayed in Fig. 12e. There is little or no work done on ions in the electron diffusion region around the $x$-line. This is consistent with tail measurements by Angelopoulos et al. (2013). With the work done on the ion current as a measure, the ion diffusion region extends from just past the $x$-line to the pile-up regions. Figure $12 \mathrm{f}$ shows the work done by $\mathbf{E}$ on the total current, $\mathbf{J}=\mathbf{J}_{e}+\mathbf{J}_{e}$. This work shows up in the radiation transport in terms of changes in magnetic energy and Poynting flux, as will be discussed in a later section.

There is a significant discrepancy between the magnitude of $\mathbf{J} \cdot \mathbf{E}$ in Fig. $12 \mathrm{f}$ near the pile-up front and the magnitude of $D_{e}$ in Fig. $12 \mathrm{~d}$ near the pile-up front. To explain this, we define $D_{e}$ as

$$
\begin{aligned}
& D_{e} \approx \mathbf{J} \cdot \mathbf{E}-W_{\mathrm{adv}} \\
& W_{\mathrm{adv}} \equiv-\mathbf{J} \cdot\left(\mathbf{v}_{e} \times \mathbf{B}\right)
\end{aligned}
$$

Here $W_{\text {adv }}$ can be interpreted as work done on the total current, $\mathbf{J}$, by the advective part of $\mathbf{E}$ in the electron fluid. This work is subtracted from $D_{e}$. In addition to Eq. (9b), there are three other useful equivalent expressions for $W_{\text {adv }}$

$$
\begin{aligned}
& W_{\mathrm{adv}}=-n e \mathbf{B} \cdot\left(\mathbf{v}_{e} \times \mathbf{v}_{i}\right) \\
& W_{\mathrm{adv}}=-\mathbf{J} \cdot\left(\mathbf{v}_{i} \times \mathbf{B}\right) \\
& W_{\mathrm{adv}}=-\mathbf{J} \cdot(\mathbf{U} \times \mathbf{B}), \quad \mathbf{U}=\mathbf{U}_{\mathrm{MHD}} \equiv \frac{m_{e} \mathbf{v}_{e}+M_{i} \mathbf{v}_{i}}{m_{e}+M_{i}}
\end{aligned}
$$

The first and second expressions are obtained from Eq. (9b) by using the equality $\mathbf{J}=$ $e n\left(\mathbf{v}_{i}-\mathbf{v}_{e}\right.$ ) to substitute for $\mathbf{J}$ (yielding Eq. (9c)), or to substitute for $\mathbf{v}_{e}$ (yielding Eq. (9d)). Equations (9e), is derived by multiplying (9b) by the electron mass, $m_{e}$, and (9d) by the ion mass, $M_{i}$, and adding. The physical interpretation of Eqs. (9b)-(9d) in terms of the electron or ion fluids is not apparent because they employ both electron and ion fluid velocities. By contrast, Eq. (9e) makes reference only to the MHD variables $\mathbf{J}$ and $\mathbf{U}$ so that $W_{\text {adv }}$ can be interpreted as the work done by the advective, ideal-MHD part of $\mathbf{E}$.

Equation (9c) shows that $\left|W_{\text {adv }}\right|$, can become large in regions where $\left(\mathbf{v}_{e} \times \mathbf{v}_{i}\right)$ has a significant component parallel to $\mathbf{B}$, such as near the pile-up front, where $\mathbf{B}$ is in the $y$-direction, $\mathbf{v}_{e}$ is mainly in the $x$-direction and $\mathbf{v}_{i}$ has a significant component in the $z$-direction. It is also relatively large throughout the central exhaust except in the neighborhood of the $x$-point.

It is interesting to compare $D_{e}$ as a measure of MHD-fluid diffusion regions with $\mathbf{J}_{e} \cdot \mathbf{E}$ and $\mathbf{J}_{i} \cdot \mathbf{E}$ as separate measures of electron and ion diffusion regions. In the ion (or electron) single-fluid there is no difference between $\mathbf{J}_{s} \cdot \mathbf{E}$ and $\mathbf{J}_{s} \cdot\left(\mathbf{E}+\mathbf{v}_{s} \times \mathbf{B}\right)$ because $\mathbf{J}_{s}=q_{s} n_{s} \mathbf{v}_{s}$, where $s=e$ or $i$. This means that $\mathbf{J}_{s} \cdot \mathbf{E}=\mathbf{J}_{s} \cdot \mathbf{R}_{s}$ from Ohm's Law (Eqs. (3a), (3b)). In a resistive single-species-fluid with pressure and inertia ignored, $\mathbf{R}_{s}=-\eta_{s} \mathbf{J}_{\mathrm{s}}$, so $\mathbf{J}_{s} \cdot \mathbf{E}=$ 

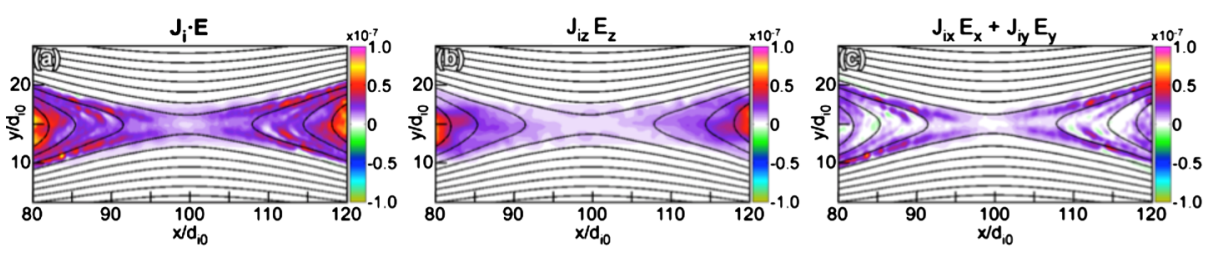

Fig. 13 (a) Work $\mathbf{j}_{i} \cdot \mathbf{E}$ performed on ions by $\mathbf{E}$-field (b) and (c), work decomposed into two contributions to the scalar product: (b) $\left(J_{i z} E_{z}\right)$, the part of the work performed by the out-of-plane electric field, $E_{z}$; (c) the term, $\left(J_{i x} E_{x}+J_{i y} E_{y}\right)$, plotted in Fig. $12 \mathrm{c}$, is the part of the work performed by in-plane components of $E$-mainly due to the Hall electric field

$-\eta_{s}\left|\mathbf{J}_{s}\right|^{2}$, which says that the work on $\mathbf{J}_{s}$ is dissipated as Joule heating through the resistivity, $\eta_{s}$, of species $s$, consistent with a collisionally-resistive magnetic diffusion region for that species. By adding Eq. (3a) times the electron mass to Eq. (3b) times the ion mass one obtains the MHD version of the generalized Ohm's law, $\mathbf{E}+\mathbf{U} \times \mathbf{B}=\mathbf{R}_{\mathrm{MHD}}=\left(m_{e} R_{e}+\right.$ $\left.M_{i} R_{i}\right) /\left(m_{e}+M_{i}\right)$, so that $D_{e}=\mathbf{J} \cdot \mathbf{R}_{\mathrm{MHD}}$. In a resistive single-fluid with pressure and inertia ignored, $\mathbf{R}_{\mathrm{MHD}}=\eta_{\mathrm{MHD}} \mathbf{J}$, so $D_{e}=\eta_{\mathrm{MHD}}|\mathbf{J}|^{2}$ which says that the work on $\mathbf{J}$ is dissipated as Joule heating, consistent with a resistive MHD diffusion region.

Hence the MHD-single-fluid "diffusion region" in a collisionless plasma is not simply the sum (Fig. 12f) of the electron-fluid and ion-fluid diffusion regions shown in Figs. 12a and 12e. Instead, it is given by $D_{e}$ (Fig. 12d) which shows that the collisionless MHD diffusion region coincides with the electron diffusion region in the neighborhood of the $x$-line.

In Fig. 13 the scalar work performed by the electric field on ions is broken into component contributions:

$$
\mathbf{J}_{i} \cdot \mathbf{E}=\left(J_{i z} E_{z}\right)+\left(J_{i x} E_{x}+J_{i y} E_{y}\right)
$$

The term $\left(J_{i z} E_{z}\right)$ plotted in Fig. 13b is the part of the work performed by the out-of-plane electric field, $E_{z}$, and the term $\left(J_{i x} E_{x}+J_{i y} E_{y}\right)$ plotted in Fig. $13 \mathrm{c}$, is the part of the work performed by in-plane components of $\mathbf{E}$-mainly due to the Hall electric field. The sum, $\mathbf{J}_{i} \cdot \mathbf{E}$, is plotted in Fig. 13a, which should be compared with Fig. 12e. In Fig. 12e the work is unsmoothed whereas in Fig. 13 the work is smoothed. Smoothing improves image visibility and reduces noise but also reduces peak values, replacing them with average values. Once again we see that very little work is performed on ions in the electron diffusion region.

The work done by the Hall electric field which dominates in Fig. 13c, is concentrated around the separatrices with regions of less work around the central exhaust while the work done by the out-of-plane (reconnection) electric field dominates around the central exhaust (Fig. 13b). Work on ions performed by the Hall field near the separatrices has also been found in reconnection experiments on the laboratory device, MRX, at Princeton (Yoo et al. 2013). A plot of the Hall work in the reconnection plane in Yoo et al. (2013) reveals a gap in the central exhaust similar to that in Fig. 13c. From Fig. 13a it is clear that the work done by the Hall field and the work done by the reconnection field fit together with little overlap, producing a smooth ion diffusion region in the entire exhaust from $x$-line to pileup. However, recent measurements in the tail (Eastwood et al. 2015) appear to show a large frozen-in ion region rather than a large ion diffusion region upstream of a dipolarization front. There are several possible explanations for this discrepancy. One is that present-day reconnection simulations do not run for long enough times. They need to be run for much longer times, enabling the pile-up fronts to move much further from the $x$-line than the 23 Harris ion inertial lengths, $d_{i 0}$, in Simulations $\mathrm{A}$ and $\mathrm{B}$ at time $t \Omega_{i}=30$. Preliminary bigger and longer-time simulations with mass ratio 25 appear to show the reappearance of a large 
Fig. 14 (a) Work, $-\mathbf{J} \cdot \mathbf{E}$, performed by the total current on electric fields results in (b) divergence of Poynting flux, $\nabla \cdot \mathbf{S}$, and (c) time rate-of-change of (mainly) magnetic field energy density (see Eq. (7a)). Striations in the inflow are electromagnetic whistler waves (Goldman et al. 2014)

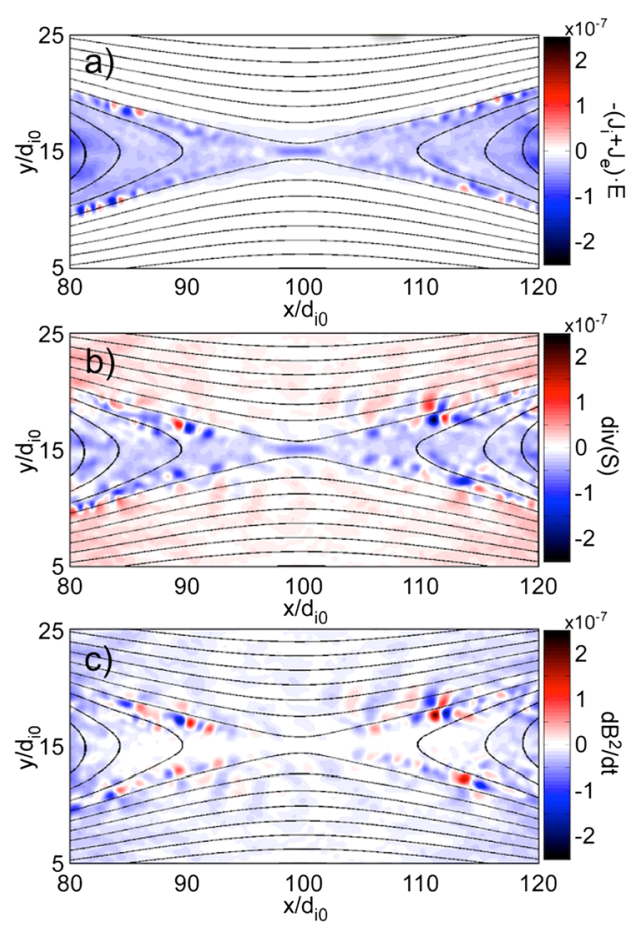

frozen-in ion diffusion region upstream of the front Another possible explanation is that the uniform thin-current-sheet model of the cross-tail current sheet needs to be replaced in future simulations by a current sheet which is significantly thicker downstream where the pile-up front butts up against it.

\subsection{Simulation Results: Field Energy Transport and Diffusion Regions}

In Fig. 14 we interpret the results of the $\mathbf{J} \cdot \mathbf{E} \neq 0$ condition for a diffusion region in terms of the electromagnetic field energy transport processes in Eq. (7a). All images in this figure are again smoothed, so that the local magnitudes of the terms in Eq. (7a) plotted in Fig. 14 are averages over the magnitudes of the unsmoothed terms (e.g., in Fig. 12f). Almost all of the work $-\mathbf{J} \cdot \mathbf{E}$, performed by the total current on the field in Fig. 14a is negative. In the exhaust this work results in the negative values of the divergence of the Poynting flux in Fig. 10b, signifying that Poynting flux is delivered into the exhaust. From Fig. 10b, 10c it is evident that some of this flux comes from the inflow region, where $\nabla \cdot \mathbf{S}$ is positive and balanced by magnetic energy density which is decreasing with time (i.e., no work is performed in the inflow region). Most of the divergence of the Poynting flux, $\nabla \cdot \mathbf{S}$, in both regions is associated with $\partial_{y} S_{y}$ (not shown) which changes sign across the separatrix. There is also a significant Poynting flux component, $S_{x}$, in the outflow direction along the separatrix legs, as in Fig. 14b. This is mostly Hall Poynting flux, $\left(\mathbf{E}_{H} \times \mathbf{B}_{H}\right)_{x}$ (Shay et al. 2011). It doesn't show up too well in the divergence because $\left|\partial_{x} S_{x}\right| \ll\left|\partial_{y} S_{y}\right|$. Figure $14 \mathrm{c}$ illustrates that magnetic energy density is weakly decreasing in time far from the $x$-point in both inflow regions and in the exhausts. Since there is no work performed in the inflow region, the decreasing magnetic energy there is associated with Poynting flux, $S_{y}$, formed in the inflow region (where $\partial_{y} S_{y}>0$ ) and deposited in the exhaust region (where $\partial_{y} S_{y}<0$ ). 
The time rate of change of magnetic energy density in the exhaust is small except near the front. Although not shown in Fig. 14, magnetic energy is carried downstream by $S_{x}$ and isn't dumped until downstream of the dipolarization front, where it is deposited in the flanks of the still untorn current sheet.

A striking feature is present in Figs. 14b, 14c: white bands almost perpendicular to $B$ field lines in the inflow region. Recently it has been shown that these bands are the phase fronts of almost parallel-propagating EM whistler waves generated by electron phase space holes on separatrix legs (Goldman et al. 2014). Such whistler waves are interesting for at least two reasons: They can act as a signal that a spacecraft is in the inflow region near the $x$-line, and they produce $15 \%$ modulations in the reconnection rate, as measured by the reconnection field, $E_{z}$ in the 2D PIC simulations depicted here.

\subsection{Simulation Results: Particle Energy Transport and Diffusion Regions}

Figure 15 is a visualization of the electron and ion energy transport processes associated with the $\mathbf{J}_{s} \cdot \mathbf{E}$ work per unit volume measure of the species $s=e, i$ diffusion regions discussed earlier. The particle transport equations are given by Eq. (7b). The energy density is $U_{s}=n_{s}\left\langle m_{s} v^{2} / 2\right\rangle=n_{s} \int d^{3} v f_{s}(\mathbf{v})\left(m_{s} v^{2} / 2\right)$, where the particle distribution function, $f_{s}(\mathbf{v})$ is normalized to one. Note that with the substitution, $\mathbf{v}=\mathbf{u}_{s}+\delta \mathbf{v}_{s}$, where $\mathbf{u}_{s}=\langle\mathbf{v}\rangle_{s}, U_{s}$ splits into the particle bulk flow energy density, $m_{s} \mathbf{u}_{s}^{2} / 2$, and the thermal energy density, $n\left\langle m_{s}(\delta v)^{2} / 2\right\rangle$. Positive work, $\mathbf{J}_{s} \cdot \mathbf{E}$ in the particle transport Eq. (7b), shows up as a net positive $\left(\partial_{t} U_{s}+\nabla \cdot \mathbf{Q}_{\mathbf{s}}\right)$, where $\mathbf{Q}_{\mathbf{s}}$ is the total electron energy flux, $\mathbf{Q}_{s}=n\left\langle\mathbf{v} m_{s} v^{2} / 2\right\rangle$. With the substitution, $\mathbf{v}=\mathbf{u}_{s}+\delta \mathbf{v}_{s}, \mathbf{Q}_{\mathbf{s}}$ becomes a sum of electron energy fluxes: the bulk electron energy flux, the enthalpy flux, and the electron heat flux, defined in Eqs. (8a)-(8e). Figure 15d shows that the divergence of the heat flux is essentially negligible.

Although it is clear from the left column in Fig. 15 that work is performed on electrons in the inner electron diffusion region in the vicinity of the $x$-line (purple region near $x=$ $100 d_{i 0}$ ), there is virtually no positive $\partial_{t} U_{e}$, in the entire exhaust (except for holes on the separatrices). Between $x=115 d_{i 0}$ and the beginning of the as-yet-unreconnected cross-tail current sheet near $x=125 d_{i 0}$, in the vicinity of the central exhaust, $\partial_{t} U_{e}$ is everywhere negative and there is outgoing $\mathbf{Q}_{e}$ flux ( $\left.\operatorname{div} \mathbf{Q}_{e}>0\right)$ and positive work in the central exhaust. The positive work in the electron diffusion region and in the exhaust is therefore directly converted into outgoing electron energy density flux $\left(\nabla \cdot \mathbf{Q}_{e}>0\right)$. This flux is not deposited $\left(\nabla \cdot \mathbf{Q}_{e}<0\right)$ until beyond $x=125 d_{i 0}$, i.e., beyond the pile up front (see green regions in Fig. 15c. This will again be shown in conjunction with Fig. 17 later.

Kinetic electron distributions in the region identified as the electron diffusion region here are agyrotropic, consistent with definition (iv) of the electron diffusion region. High resolution determination of electron distribution functions on MMS should be able to use this signature of the electron diffusion region. This will be the subject of another paper.

The right column in Fig. 15 exhibits corresponding ion energy transport processes associated with the $\mathbf{J}_{i} \cdot \mathbf{E}$ work per unit volume measure of the ion diffusion region. The integrated ion work is significantly larger in magnitude than the integrated electron work.

The right column in Fig. 15 confirms that positive work is performed on ions by the Hall and reconnection fields throughout the exhaust and beyond, as shown earlier in the signed$\log$ plot, Fig. 12. The work on ions remains positive into and through the pile-up region at $x=123 d_{i 0}$ near the central exhaust and beyond, up to the ambient unreconnected Harris sheet. In that unreconnected thin ("cross-tail") Harris sheet there are turbulent-looking patches of positive to negative reversals in all transport terms as well as $\mathbf{J}_{i} \cdot \mathbf{E}$. These may be associated with incipient secondary reconnection or with instability. 

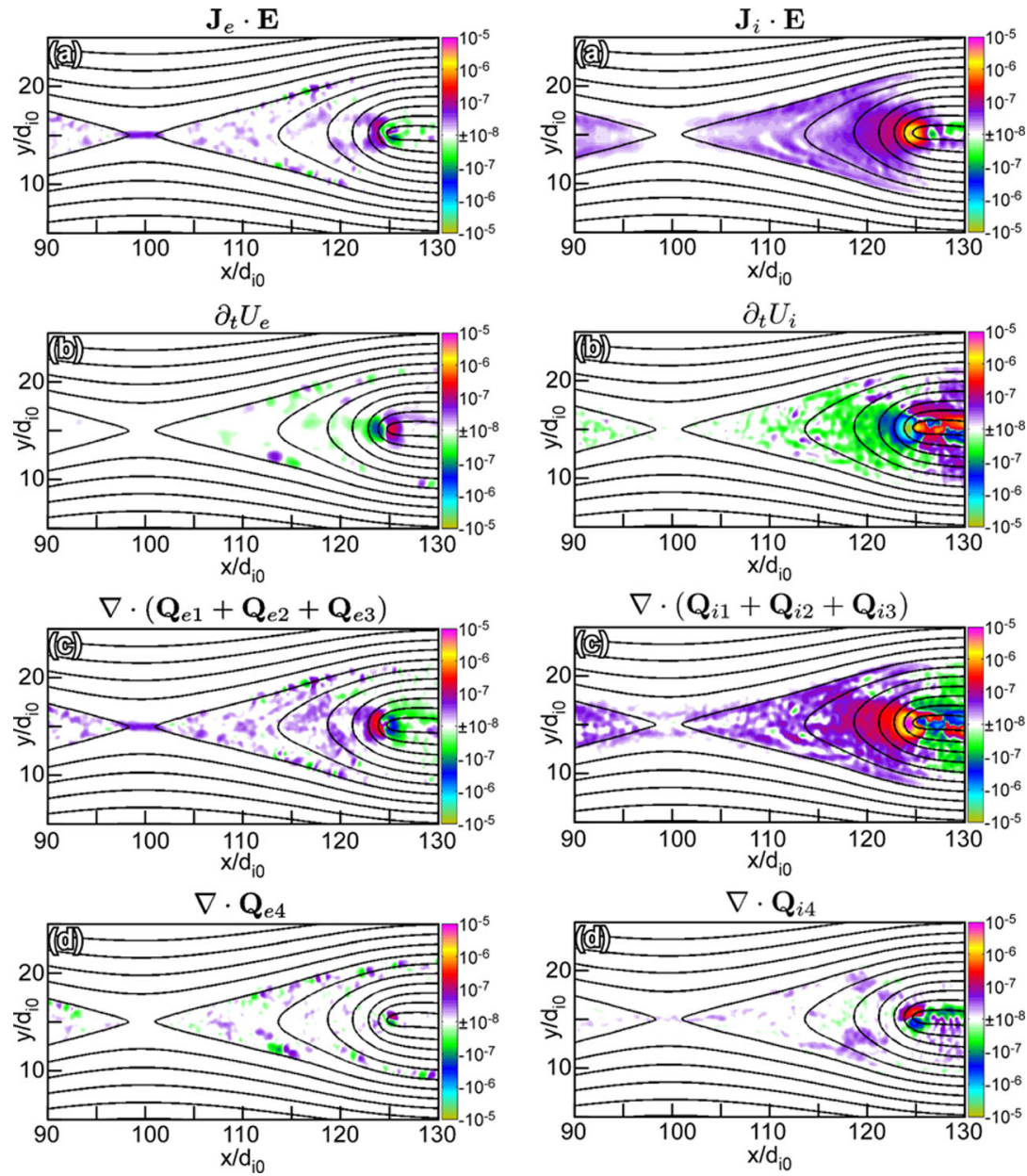

Fig. 15 Terms in particle transport equations ((7b) and (8a)-(8e)). Signed logarithmic scale. Left column shows electron transport terms and right column shows ion transport terms. (a) Work done by electric field on electron/ion current. (b) Time derivative of total electron/ion energy density, $U_{e}$ (includes both coherent and thermal energy densities). (c) Divergence of electron/ion energy bulk energy flux, $Q_{s} 1$ plus two enthalpy flux terms, $Q_{s 2}+Q_{s 3}$. (d) Divergence of heat flux, $Q_{s 4}=q_{s}$

Both $\partial_{t} U_{i}$ and $\nabla \cdot \mathbf{Q}_{i}$, which were negative and positive, respectively throughout the exhaust (where work is performed) finally turn positive and negative, respectively in the flanks of the unreconnected current, region where no work is performed (Fig. 15a). The flanks of the unreconnected thin Harris sheet at both higher and lower $y$-locations experience flow from the exhaust in this and other 2D simulations initiated with uniformly thin Harris sheets. Ion transport in the flanks might be altered for very long runs, for 3D simulations, for simulations with thicker initial cross-tail current sheets and/or for simulations which include Earth's dipole magnetic field.

There is a lesson here for MMS. The Poynting flux, $\mathbf{S}$ and the particle fluxes, $\mathbf{Q}_{s}$, should be measured in the exhaust, in the pile-up (dipolarization) front and in the unreconnected cross-tail current sheet beyond the front! These are emminently measurable quantities. In 

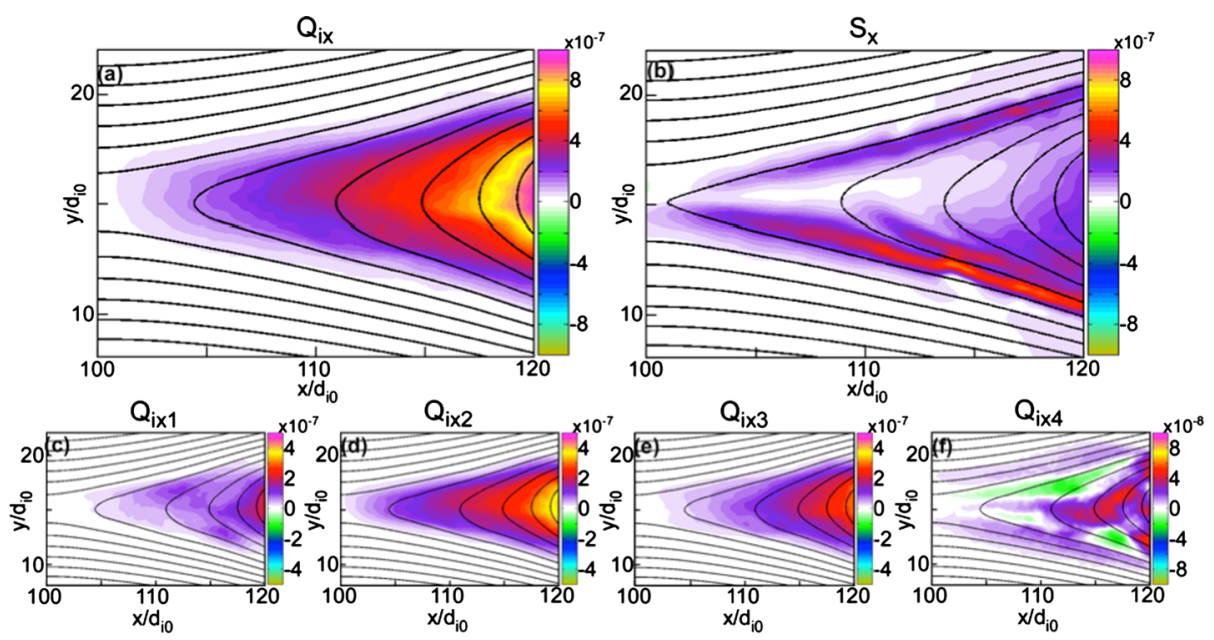

Fig. 16 Ion energy flux, $Q_{i x}$ compared to Poynting flux, $S_{x}$ from Simulation A. (a) $Q_{i x}$, (b) $S_{x}$, (c) $Q_{i 1 x}$, (d) $Q_{i x 2}$, (e) $Q_{i x 3}$, (f) $Q_{i x 4}=q_{i}$

fact, both $S_{x}$ and the entropy contribution, $Q_{i 2 x}+Q_{i 3 x}$ to the total ion energy flux (Eq. (8a)(8e)), $Q_{i x}$, have already been studied statistically and compared using Cluster data in exhaust regions (Eastwood et al. 2013), but not in pile-up regions or beyond. The Cluster studies show that $S_{x}$ tends to dominate along the separatrices and that the entropy contribution, $Q_{i 2 x}+Q_{i 3 x}$ dominates along the central exhaust. These measurements are completely consistent with findings from the present 2D simulations shown in Fig. 16 for $S_{x}$ and $Q_{x}$ and especially for the contribution to $Q_{x}$ corresponding to enthalpy flux. The large Poynting flux along the separatrix has also been noticed in PIC simulations and has been attributed (Shay et al. 2011) to kinetic Alfven wave flux said to be sufficiently intense to reach the auroral ionosphere.

Space-integrated transport quantities for fields and ions are depicted in Fig. 17 which provides an additional way to understand the transport in Fig. 15.

In Fig. 17a the $y$-integrated values of the following quantities are plotted as functions of $x$, starting at the $x$-point: work/vol on ions (blue), work/vol on electrons (green), negative time rate of change of magnetic (red) and electric (cyan) energy densities, divergence of the Poynting flux, $\boldsymbol{\nabla} \cdot \mathbf{S}$ (magenta), and $\mathbf{J} \cdot \mathbf{E}+\nabla \cdot \mathbf{S}$ (yellow). A vertical dashed line is drawn at $x=125 d_{i 0}$, slightly downstream from the pile-up front. The integrated time rateof-change of electric energy density is negligible compared to the integrated rate of change of magnetic energy density and the work on electrons is negligible compared to the work on ions. All other $y$-integrated transport terms increase with $x$ until the pile-up front, where they drop precipitously (and $\nabla \cdot \mathbf{S}$ goes negative). At larger $x, y$-integrated Poynting flux is deposited and the $y$-integrated time-rate-of-change of magnetic energy density begins to increase, consistent with Fig. 15.

In Fig. 17b the results of an additional cumulative integral over $x$ beginning at the $x$ point for each of the transport terms are displayed. The cumulative rate of loss of integrated magnetic energy density increases until the pile-up front where the loss begins to decrease. The cumulative integrated work on ions increases until just after the pile-up front where it plateaus at a value almost ten times that of the cumulative integrated work on electrons.

In Figs. $17 \mathrm{c}$ and $17 \mathrm{~d}$, integrated ion transport equation terms are plotted vs. $x$. Once again there is an important transition around the location of the pile-up front. The $y$-integrated 

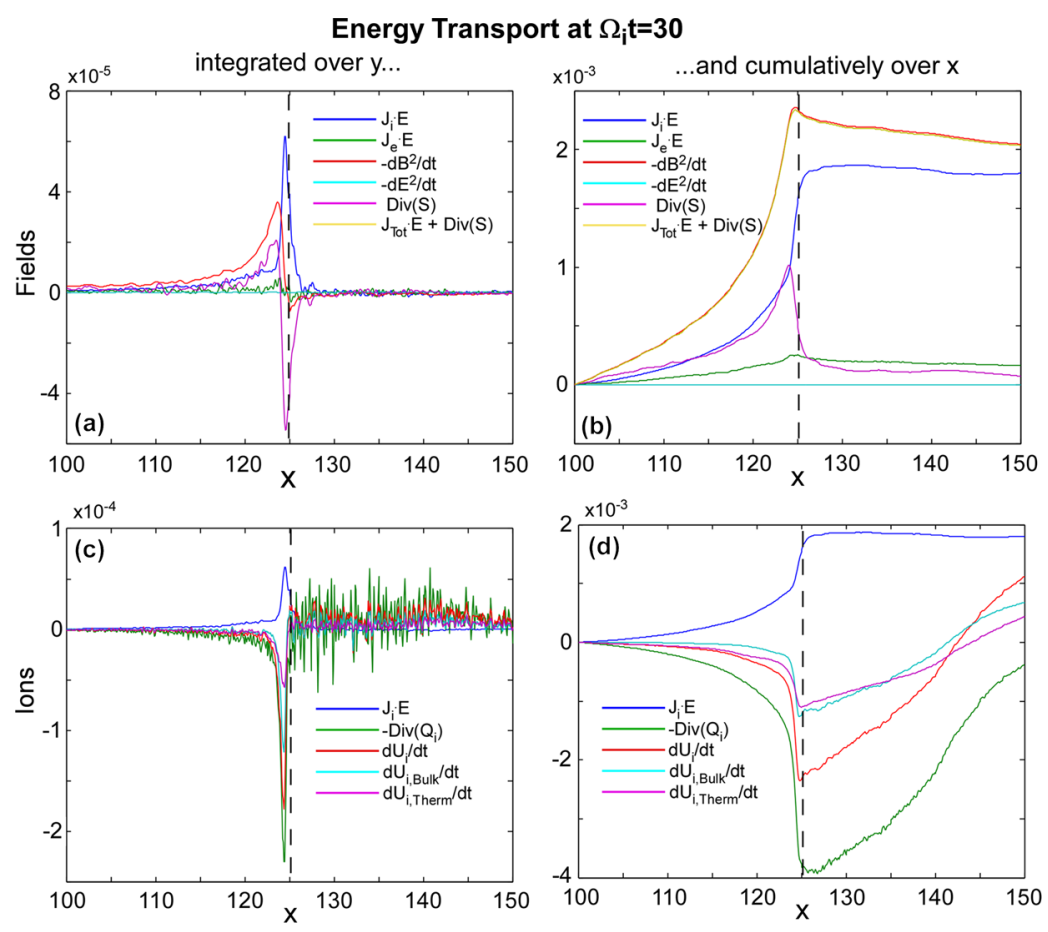

Fig. 17 (a) and (b): Integrated terms in field transport equation and (c) and (d) in ion transport equation. Integrated over $y$ alone in (a) and (c). Integrated over $y$ and cumulatively integrated over $x$ in (b) and (d). See labels for line-plots of different colors

$\boldsymbol{\nabla} \cdot \mathbf{Q}_{i}$ swings up from decreasing to increasing at the front, oscillating between positive and negative downstream of the front. The $y$-integrated $\partial_{t} U_{i}$ behaves similarly, but with positive oscillations downstream of the front. In Fig. 17d the plotted ion transport terms are integrated over $y$ and cumulatively integrated in $x$ from the $x$-point. The integrals over $\partial_{t} U_{i}$ are decomposed into bulk and thermal parts which are roughly equal to each other at all $x$. Both decrease from $x=100 d_{i 0}$ to $125 d_{i 0}$ (just upstream of the front) and increase after $x=125 d_{i 0}$ (downstream of the front), turning positive at around $x=140 d_{i 0}$.

We note that recent simulations by Birn and Hesse (2014) are in accord with some of our transport results. They carried out 2D PIC simulations of magnetotail dynamics with an initial 2-D current sheet to compare with their MHD simulations. Their focus is on the onset of reconnection and subsequent energy transfer and conversion together with entropy studies. Their 2D PIC simulation is perhaps more realistic for Earth's magnetotail since they use a 2-D initial current sheet but they also employ a smaller simulation box and have less separation between their reconnection front and $x$-point. They use a mass ratio of 100 . Interestingly, they find some of the same results that we find from our less sophisticated 1-D Harris current sheet simulations in a larger box with mass ratio of 256, suggesting there is merit in the Harris sheet initiation method. They find a small contribution to the work done on electrons by the electric field at the pile-up front, barely visible on the left in their Fig. 19, whereas we find significant $\mathbf{J}_{e} \cdot \mathbf{E}$ work on electrons in a narrow region in the pile-up front. Their transport studies show the conversion of inflowing magnetic flux into outflowing particle energy flux (mainly enthalpy flux) as we find also. However their particle energy transport equations are based on a single-fluid (i.e., MHD) model with a scalar pressure 
rather than on kinetic theory. Hence, they do not study electron energy transport as we do, for example, in our Fig. 15.

\section{Summary of New Results Deduced from 2D Implicit PIC Simulations of Magnetotail Reconnection Initiated by a Thin Harris Sheet with a Small Guide Field}

- The role of particle slippage, flux-non-conservation, work and kinetic features in the determination of particle diffusion regions and in their relation to each other has been explored in detail. Realization of the central goal of the MMS mission-identification of particle diffusion regions - should be expedited in the tail by an understanding of the multiple (and often different) signatures of diffusion regions found in these simulations.

- Certain features in the vicinity of (Themis) measured dipolarization fronts (DFs) are reasonably well-described by these 2D simulations. These features include the thickness of the DF, the magnetic flux pile-up, the maximum in the local reconnection electric field, the density profile and the balance between particle and magnetic pressure at the front. Features that are not well described by the simulation are the measured thick current sheet and the direction of increase of the flow velocity, $u_{x}$ (increasing upstream in Themis data, downstream in the simulation).

- The ion diffusion region (Hall field region) extends from the $x$-point to the DF at a time in the simulations at which the DF is on the order of 30 ion inertial lengths from the $x$ point. This result may change for longer runs with thicker current sheets or in $3 \mathrm{D}$. The simulation result for the work on ions, $\mathbf{J}_{i} \cdot \mathbf{E}$, is in agreement with laboratory reconnection experiments (Yoo et al. 2013). The $\mathbf{J} \cdot \mathbf{E}$ work performed on $\mathbf{J}$ by the total field, $\mathbf{E}$ looks similar to $\mathbf{J}_{i} \cdot \mathbf{E}$ in the central exhaust but receives no contribution from the Hall part of $\mathbf{E}$. The $\mathbf{J} \cdot \mathbf{E}$ work is mainly due to the advective term in the Ohm's law expression for $\mathbf{E}$. Therefore the work measure of Zenitani et al. (2011), $D_{i} \approx \mathbf{J} \cdot\left(\mathbf{E}+\mathbf{v}_{i} \times \mathbf{B}\right)=\mathbf{J} \cdot \mathbf{R}_{i}$ is not large in the central exhaust region away from the electron diffusion region.

- The physical magnitude (in $\mathrm{mV} / \mathrm{m}$ and in $\mathrm{nT}$ ) of electric and magnetic fields found in simulations agrees well with measurements even when artificial mass ratios are used in the simulations, provided one interprets appropriate particle mass (electron or ion) as physical.

- In the region between the $x$-point and the DF magnetic energy is carried by Poynting flux (mainly along the separatrices) and non-bulk ion energy carried by ion enthalpy flux (mainly along the neutral line), in agreement with measurements of Eastwood et al. (2013). Both theory and measurement reveal the ion heat flux to be much smaller in magnitude than the ion enthalpy flux.

- Transport analysis shows that Poynting flux, $S_{x}$, deposits magnetic energy and ion energy flux, $Q_{x}$ deposits ion energy in a region where no work is performed-on the flanks of the remaining high-density thin current sheet just downstream of the dipolarization front (DF). In this region both $\partial_{t} B^{2}$ and $\partial_{t} U_{i}$ are positive. It will be interesting to see if this result holds for $2 \mathrm{D}$ simulations with thicker current sheets and for $3 \mathrm{D}$ simulations.

- The dominant contribution to the electrostatic "Hall" electric field (proportional to $\mathbf{J} \times \mathbf{B}$ ) comes from the cross-product of the non-Hall current (normal to the current sheet) with the non-Hall (in-plane) magnetic field rather than from the cross-product of the Hall (inplane) current with the Hall (quadrupole) magnetic field.

The physics of magnetotail reconnection provided by 2D implicit PIC kinetic simulations with a very small but significant guide field and a mass ratio equal to or greater than 256 has 
been described in detail. New insights for understanding particle and field behaviour and transport processes have been developed which should be useful for identifying diffusion regions during the MMS mission. 2-D simulations of tail reconnection with a realistic guide field have been sufficient to secure agreement with a number of measurements. In addition these 2D simulations lay the foundation for future comparisons with more sophisticated current sheets and with 3D kinetic simulations which are just now beginning to uncover still newer physics.

Acknowledgements This work has been supported by NASA Grants NNX08AO84G and NNX12AG70G and by NASA computer resources. Thanks to the other members of our MMS-IDS team, Drs. Jack Gosling, Sterfan Eriksson and Laila Andersson for their help and insights and to Dr. S. Markidis for his role in developing the implicit PIC codes use for these simulations. Also thanks to Profs. J. Drake, R. Ergun and Dr. J. Eastwood for useful discussions.

Open Access This article is distributed under the terms of the Creative Commons Attribution License which permits any use, distribution, and reproduction in any medium, provided the original author(s) and the source are credited.

\section{References}

V. Angelopoulos, A. Runov, X.-Z. Zhou, D.L. Turner, S.A. Kiehas, S.-S. Li, I. Shinohara, Electromagnetic energy conversion at reconnection fronts. Science 341, 1478-1482 (2013). doi:10.1126/science.1236992

N. Aunai, M. Hesse, M. Kuznetsova, Electron nongyrotropy in the context of collisionless reconnection. Phys. Plasmas 20, 092903 (2013)

N. Bessho, A. Bhattacharjee, Instability of the current sheet in the Earth's magnetotail with normal magnetic field. Phys. Plasmas 21, 102905 (2014)

J. Birn, M. Hesse, Forced reconnection in the near magnetotail: Onset and energy conversion in PIC and MHD simulations. J. Geophys. Res. 119, 290 (2014)

J. Birn, J.F. Drake, M.A. Shay, B.N. Rogers, R.E. Denton, M. Hesse, M. Kuznetsov, Z.W. Ma, A. Bhattacharje, A. Otto, P.L. Pritchett, Geospace Environmental Modeling (GEM) magnetic reconnection challenge. J. Geophys. Res. 106(A3), 3715-3719 (2001)

D. Biskamp, Magnetic Reconnection in Plasmas (Cambridge University Press, Cambridge, 2000)

S.V. Bulanov, F. Pegoraro, A.S. Sakharov, Magnetic reconnection in electron magnetohydrodynamics. Phys. Fluids B 4, 2499 (1992). doi:10.1063/1.860467

C. Cattell, J. Dombeck, J. Wygant, J.F. Drake, M. Swisdak, M.L. Goldstein, W. Keith, A. Fazakerley, M. André, E. Lucek, A. Balogh, Cluster observations of electron holes in association with magnetotail reconnection and comparison to simulations. J. Geophys. Res. 110(A9), 1211 (2005). doi:10.1029/ 2004JA010519

H. Che, J.F. Drake, M. Swisdak, A current filamentation mechanism for breaking magnetic field lines during reconnection. Nature 474, 184-187 (2011). doi:10.1038/nature10091

B. Coppi, G. Laval, R. Pellat, Dynamics of the Geomagnetic Tail. Phys. Rev. Lett. 16 (1966)

W. Daughton, V. Roytershteyn, H. Karimabadi, L. Yin, B.J. Albright, B. Bergen, K.J. Bowers, Nat. Phys. (2011). doi:10.1038/NPHYS1965

W. Daughton, T.K.M. Nakamura, H. Karimabadi, V. Roytershteyn, B. Loring, Computing the reconnection rate in turbulent kinetic layers by using electron mixing to identify topology. Phys. Plasmas 21, 052307 (2014). doi:10.1063/1.4875730

X. Deng, M. Ashour-Abdalla, M. Zhou, R. Walker, M. El-Alaoui, V. Angelopoulos, R.E. Ergun, D. Schriver, Wave and particle characteristics of earthward electron injections associated with dipolarization fronts. J. Geophys. Res. 115, A09225 (2010). doi:10.1029/2009JA015107

J.F. Drake, Y.C. Lee, Kinetic theory of tearing instabilities. Phys. Fluids 20, 1341-1353 (1977). doi:10.1063/ 1.862017

J.F. Drake, R.G. Kleva, M.E. Mandt, Structure of thin current layers: implications for magnetic reconnection. Phys. Rev. Lett. 73, 1251-1254 (1994)

J.F. Drake, M. Swisdak, C. Cattell, M.A. Shay, B.N. Rogers, A. Zeiler, Formation of electron holes and particle energization during magnetic reconnection. Science 299, 873-877 (2003). doi:10.1126/ science. 1080333 
J.F. Drake, M.A. Shay, M. Swisdak, The Hall fields and fast magnetic reconnection. Phys. Plasmas 15, 042306 (2008). doi:10.1063/1.2901194

J.F. Drake, M. Swisdak, P.A. Cassak, T.D. Phan, On the 3-D structure and dissipation of reconnection-driven flow bursts. Geophys. Res. Lett. (2014). doi:10.1002/2014GL060249

J.P. Eastwood, T.D. Phan, M. Øieroset, M.A. Shay, Average properties of the magnetic reconnection ion diffusion region in the Earth's magnetotail: 2001-2005 Cluster observations and comparison with simulations. J. Geophys. Res. 115, A08215 (2010a). doi:10.1029/2009JA014962

J.P. Eastwood, M.A. Shay, T.D. Phan, M. Øieroset, Asymmetry of the ion diffusion region Hall electric and magnetic fields during guide field reconnection: observations and comparison with simulations. Phys. Rev. Lett. 104(20), 205001 (2010b). doi:10.1103/PhysRevLett.104.205001

J.P. Eastwood, T.D. Phan, J.F. Drake, M.A. Shay, A.L. Borg, B. Lavraud, M.G.G.T. Taylor, Energy partition in magnetic reconnection in Earth's magnetotail. Phys. Rev. Lett. 110 (2013). ISSN:0031-9007

J.P. Eastwood, M.V. Goldman, H. Hietala, D.L. Newman, R. Mistry, G. Lapenta, Ion reflection and acceleration near magnetotail dipolarization fronts associated with magnetic reconnection. J. Geophys. Res. 120, 511 (2015)

M. Faganello, F. Pegoraro, F. Califano, L. Marradi, Collisionless magnetic reconnection in the presence of a sheared velocity field. Phys. Plasmas 17, 062102 (2010)

M.V. Goldman, G. Lapenta, D.L. Newman, S. Markidis, H. Che, Jet deflection by very weak guide fields during magnetic reconnection. Phys. Rev. Lett. 107(13), 135001 (2011). doi:10.1103/PhysRevLett.107. 135001

M.V. Goldman, D.L. Newman, G. Lapenta, L. Andersson, J.T. Gosling, S. Eriksson, S. Markidas, J. Eastwood, R. Ergun, Cerenkov emission of quasiparallel whistlers by fast electron phase-space-holes during magnetic reconnection. Phys. Rev. Lett. 112, 145002 (2014)

M. Hamrin, O. Marghitu, P. Norqvist, S. Buchert, M. André, B. Klecker, L.M. Kistler, I. Dandouras, The role of the inner tail to midtail plasma sheet in channeling solar wind power to the ionosphere. J. Geophys. Res. 117, A06310 (2012). doi:10.1029/2012JA017707

H.E. Harris, On a plasma sheath separating regions of oppositely directed magnetic field. Nuovo Cimento 23(1), 115-121 (1962)

M. Hesse, K. Schindler, A theoretical foundation of general magnetic reconnection. J. Geophys. Res. 93, 5559-5567 (1988)

G. Hornig, K. Schindler, Magnetic topology and the problem of its invariant definition. Phys. Plasmas 3, 781 (1996)

J.D. Huba, Hall magnetic reconnection: guide field dependence. Phys. Plasmas 12, 012322 (2005)

J.D. Huba, L.I. Rudakov, Hall magnetic reconnection rate. Phys. Rev. Lett. 93, 175003 (2004). doi:10.1103/ PhysRevLett.93.175003

Yu.V. Khotyaintsev, C.M. Cully, A. Vaivads, M. Andre, C.J. Owen, Plasma jet braking: energy dissipation and nonadiabatic electrons. Phys. Rev. Lett. 106, 165001 (2011)

G. Lapenta, S. Markidis, A. Divin, M. Goldman, D.L. Newman, Scales of guide field reconnection at the hydrogen mass ratio. Phys. Plasmas 17, 082106 (2010)

G. Lapenta, S. Markidis, A. Divin, M. Goldman, D. Newman, Bipolar electric field signatures of reconnection separatrices for a hydrogen plasma at realistic guide fields. Geophys. Res. Lett. 38, L17104 (2011)

G. Lapenta, M. Goldman, D. Newman, S. Markidis, A. Divin, Electromagnetic energy conversion in downstream fronts from 3D kinetic reconnection. Phys. Plasmas 21(5), 055702 (2014)

A. Le, J. Egedal, O. Ohia, W. Daughton, H. Karimabadi, V.S. Lukin, Regimes of the electron diffusion region in magnetic reconnection. Phys. Rev. Lett. 110, 135004 (2013). doi:10.1103/PhysRevLett.110.135004

B. Lembege, R. Pellat, Stability of a thick two-dimensional quasineutral current sheet. Phys. Fluids 25, 19952004 (1982)

Y.-H. Liu, J.F. Drake, M. Swisdak, The structure of the magnetic reconnection exhaust boundary. Phys. Plasmas 19, 022110 (2012). doi:10.1063/1.3685755

Y.-H. Liu, W. Daughton, H. Karimabadi, H. Li, V. Roytershteyn, Bifurcated structure of the electron diffusion region in three-dimensional magnetic reconnection. Phys. Rev. Lett. 10, 265004 (2013)

S. Markidis, G. Lapenta, Multi-scale simulations of plasma with iPIC3D. Math. Comput. Simul. 80(7), 15091519 (2010)

S. Markidis, G. Lapenta, L. Bettarini, M. Goldman, D. Newman, L. Andersson, Kinetic simulations of magnetic reconnection in presence of a background O+ population. J. Geophys. Res. 116, A00K16 (2011). doi:10.1029/2011JA016429

W.A. Newcomb, Motion of magnetic lines of force. Ann. Phys. 9, 347-385 (1958)

T.D. Phan, J.F. Drake, M.A. Shay, F.S. Mozer, J.P. Eastwood, Evidence for an elongated ( $>60$ ion skin depths) electron diffusion region during fast magnetic reconnection. Phys. Rev. Lett. 99, 255002 (2007). doi:10.1103/PhysRevLett.99.255002 
P.L. Pritchett, Onset and saturation of guide-field magnetic reconnection. Phys. Plasmas 12(6), 062301 (2005a). doi:10.1063/1.1914309

P.L. Pritchett, Externally driven magnetic reconnection in the presence of a normal magnetic field. J. Geophys. Res. 110, A05209 (2005b). doi:10.1029/2004JA010948

P.L. Pritchett, Onset of magnetic reconnection in the presence of a normal magnetic field: Realistic ion to electron mass ratio. J. Geophys. Res. 115, A10208 (2010). doi:10.1029/2010JA015371

P.L. Pritchett, F.V. Coroniti, Three-dimensional collisionless magnetic reconnection in the presence of a guide field. J. Geophys. Res. 109, A01220 (2004). doi:10.1029/2003JA009999

P.L. Pritchett, F.V. Coroniti, Plasma sheet disruption by interchange-generated flow intrusions. Geophys. Res. Lett. 38, L10102 (2011). doi:10.1029/2011GL047527

P.L. Pritchett, F.V. Coroniti, Structure and consequences of the kinetic ballooning/interchange instability in the magnetotail. J. Geophys. Res. 118, 146-159 (2013). doi:10.1029/2012JA018143

A. Runov, V. Angelopoulos, M.I. Sitnov, V.A. Sergeev, J. Bonnell, P. McFadden, D. Larson, K.-H. Glassmeier, U. Auster, THEMIS observations of an earthward-propagating dipolarization front. Geophys. Res. Lett. 36, L14106 (2009). doi:10.1029/2009GL038980

K. Schindler, M. Hesse, J. Birn, Magnetic field-aligned electric potentials in nonideal plasma flows. Astrophys. J. 380, 293-301 (1991)

J. Scudder, W. Daughton, "Illuminating" electron diffusion regions of collisionless magnetic reconnection using electron agyrotropy. J. Geophys. Res. 113, A06222 (2008). doi:10.1029/2008JA013035

M.A. Shay, J.F. Drake, B.N. Rogers, R.E. Denton, Alfvenic collisionless magnetic reconnection and the Hall term. J. Geophys. Res. 106, 3759 (2001)

M.A. Shay, J.F. Drake, M. Swisdak, Two-scale structure of the electron dissipation region during collisionless magnetic reconnection. Phys. Rev. Lett. 99, 155002 (2007). doi:10.1103/PhysRevLett.99.155002

M.A. Shay, J.F. Drake, J.P. Eastwood, T.D. Phan, Super-Alfvénic propagation of substorm reconnection signatures and Poynting flux. Phys. Rev. Lett. 107(6), 065001 (2011). doi:10.1103/PhysRevLett. 107.065001

M.I. Sitnov, K. Schindler, Tearing stability of a multiscale magnetotail current sheet. Geophys. Res. Lett. 37, L08102 (2010). doi:10.1029/2010GL042961

M.I. Sitnov, M. Swisdak, A.V. Divin, Dipolarization fronts as a signature of transient reconnection in the magnetotail. J. Geophys. Res. 114, A04202 (2009). doi:10.1029/2008JA013980

M.I. Sitnov, N. Buzulukova, M. Swisdak, V.G. Merkin, T.E. Moore, Spontaneous formation of dipolarization fronts and reconnection onset in the magnetotail. Geophys. Res. Lett. 40, 22 (2013)

D.A. Uzdensky, R.M. Kulsrud, Physical origin of the quadrupole out-of-plane magnetic field in hall magnetohydrodynamic reconnection. Phys. Plasmas 13, 062305 (2006)

A.E. Vapirev, G. Lapenta, A. Divin, S. Markidis, P. Henri, M. Goldman, D. Newman, Formation of a transient front structure near reconnection point in 3-D PIC simulation. J. Geophys. Res. Space Phys. 118, 14351449 (2013). doi:10.1002/jgra.50136

V.M. Vasyliunas, Nonuniqueness of magnetic field line motion. J. Geophys. Res. 77, 6271 (1972)

V.M. Vasyliunas, Theoretical models of magnetic field line merging, I. Rev. Geophys. Space Phys. 13, 303336 (1975)

J.R. Wygant, C.A. Cattell, R. Lysak, Y. Song, J. Dombeck, J. McFadden, F.S. Mozer, C.W. Carlson, G. Parks, E.A. Lucek, A. Balogh, M. Andre, H. Reme, M. Hesse, C. Mouikis, Cluster observations of an intense normal component of the electric field at a thin reconnecting current sheet in the tail and its role in the shock-like acceleration of the ion fluid into the separatrix region. J. Geophys. Res. 110(A9), 9206 (2005). doi:10.1029/2004JA010708

M. Yamada, H. Ji, T. Carter, R. Kulsrud, F. Trintchouk, Experimental investigation of the neutral sheet profile during magnetic reconnection. Phys. Plasmas 7, 1781 (2000)

M. Yamada, R. Kulsrud, H. Ji, Magnetic reconnection. Rev. Mod. Phys. 82, 603-664 (2010)

J. Yoo, M. Yamada, H. Ji, C.E. Myers, Observation of ion acceleration and heating during collisionless magnetic reconnection in a laboratory plasma. Phys. Rev. Lett. 110, 215007 (2013). doi:10.1103/ PhysRevLett.110.215007

S. Zenitani, M. Hesse, A. Klimas, M. Kuznetsova, New measure of the dissipation region in collisionless magnetic reconnection. Phys. Rev. Lett. 106, 195003 (2011). doi:10.1103/PhysRevLett.106.195003

M. Zhi-Wei, F. Shu-Ling, Generation of electric field and net charge in Hall reconnection. Chin. Phys. Lett. 25, 2934 (2008). doi:10.1088/2056-307X/25/8/055

X.-Z. Zhou, V. Angelopoulos, J. Liu, A. Runov, S.-S. Li, On the origin of pressure and magnetic perturbations ahead of dipolarization fronts. J. Geophys. Res. Space Phys. 119, 211-220 (2013). doi:10.1002/ 2013JA019394 